\title{
Attitude towards Information and non-Expected Utility Preferences: A Characterization by Choice Functions ${ }^{1}$.
}

\author{
Marc-Arthur DIAYE ${ }^{2}$ and Jean-Max KOSKIEVIC ${ }^{3}$
}

First version: $26 / 05 / 1999$

This version: $03 / 09 / 2001$

\footnotetext{
${ }^{1}$ We thank Jean-Yves Jaffray, Ed Schlee, Jean-Christophe Vergnaud and Peter Wakker for very helpful comments and remarks. We thank also the participants of seminars held at Université de Caen (GEMMA), Université d Evry, Université de Paris I and ENSAI. This paper was also presented at the CORE Summer School on Information in Games, Markets and Organizations at the Catholic University of Louvain (May 2000), the 5th International Meeting of the Society for Social Choice and Welfare in Alicante (June 2000), and the 56th ESEM in Lausanne (August 2001). A part of this research was undertaken while J.M. Koskievic was visiting ENSAI (CREST-LSM) and M.A. Diaye was visiting LIP6 (Université de Paris 6). The usual caveats of course apply.

${ }^{2}$ EPEE, Université d Evry and CREST (LSM). E-mail: marc-arthur.diaye@eco.univ-evry.fr

${ }^{3}$ EUREQua, Université Paris I. E-mail: koskievi@univ-paris1.fr
} 


\begin{abstract}
In the Allais paradox, if an agent s preferences violate independence axiom, the (nonExpected Utility) decision maker appears to be prone to dynamic inconsistency, that is in some sequential decision problem he may be expected to embark upon (action) plans which he is not going to follow through. Moreover, Wakker (1988) proves that non-EU decision maker can be made worse off, in dynamic choice setting, by getting a prior knowledge of what nature s moves will be. Thus, dynamic inconsistency and Information aversion are closely linked. Following Wakker s argument, a number of papers have set out the relationship between dynamic consistency and information attitude, but authors restrict the class of non-EU preferences by imposing different consistent properties, nonEU preferences must satisfy.

Our approach in this paper is different, instead of starting from agent s preferences to infer agent s attitude towards information, conversely we start from the attitude towards information to infer the agent s preferences logically possible. We display in the simplest dynamic version of the Allais paradox, the different possible attitudes towards information and characterize them in the Choice Functions Theory s framework. We show for instance that an agent who has non-EU preferences can be Information Averse as pointed out by Wakker (1988) but also Information Lover. Therefore, the simple observation of non-EU preferences cannot give us any piece of information about the agent s attitude towards information.
\end{abstract}

Classi cation Number: C4, D8.

Keywords: Information, Decision Theory. 


\section{Introduction}

Considering lotteries, that is to say nitely-supported probability distributions over a set of consequences (e.g., amounts of money), von Neumann \& Morgenstern (1947) introduced the independence condition for characterizing the maximization of expected utility for decision making under risk. The independence (or substitution) condition says that if a rst probabilistic mixture of a rst and a xed lottery is changed into a second probabilistic mixture by replacing the rst lottery by a second one (without changing the xed lottery), then this change is felt as an improvement if and only if the second lottery is preferred to the rst lottery. This condition, together with some other (completeness, transitivity and continuity of the preference relation) implies expected utility.

In economics, following Allais (1953) s theoretical objections against the founding principle of expected utility theory, systematic violations of the Independence axiom has been found empirically (Kahneman \& Tversky (1979)) and defended normatively (Machina (1982)). These ndings have led to alternative non-expected utility models (thereafter non-EU) during the last twenty years (See Karni \& Schmeidler (1990) for a survey).

Nevertheless, the non-EU models have been challenged in a dynamic setting because in the simplest dynamic version of the Allais paradox, if an agent s preferences violate independence axiom ${ }^{1}$, the decision maker appears to be prone to dynamic inconsistency ${ }^{2}$, that is, in some sequential decision problem he may be expected to embark upon (action) plans which he is not going to follow through. In other word, the Dynamic Inconsistent agent s actual choice upon arriving at a decision node would differ from his planned choice for that node. In such a case, the outcome of dynamic inconsistent behavior is guaranteed to be less bene cial than the outcome of an alternative course of action standing at the agent disposal ${ }^{3}$. This argument seems to demonstrate that non-EU maximizers are generically unable of behaving consistently, even in the simplest situation.

Moreover, Wakker (1988) proves that non-EU decision maker can be made worse off, in dynamic choice setting, by getting a prior knowledge of what nature s moves will be. A number of papers have set out the relationship between the independence axiom and the value of information (Wakker (1988), for instance). These papers showed in various settings that if an agent violates a version of the independence axiom, then that agent prefers less to more information. Such an agent is called Information Averse. As Machina (1989) observed, however, these arguments implicitly assume an axiom known as consequentialism , that is independence of past conterfactual events; accordingly, a more accurate statement of this nding is that a consequentialist agent who violates the Independence axiom may prefer less to more information. Finally, Karni \& Schmeidler (1991) formally demonstrated that, if the consequentialism and the reduction of compound lotteries axiom hold, then independence axiom is equivalent to dynamic consistency, a closely related

\footnotetext{
${ }^{1}$ An example of each of this argument can be constructed for any departure from Expected Utility preferences, not only in the Allais paradox framework.

${ }^{2}$ We refer to the decision-theoretic problem of dynamic inconsistent risk preferences, that is Inconsistent preferences over sequential risky decisions, in absence of time. See Caillaud \& Jullien (2000) for a discussion of the different problem of time-inconsistent preferences, that is inconsistent preferences over intertemporal decisions, in absence of risk. See also Brocas \& Carillo (2000).

${ }^{3}$ Several researchers have shown how the dynamic inconsistency argument can be adapted to make book against (that is, extract a sure payment from) a non-EU maximizer.
} 
condition to desirability of information. But consequentialism is inappropriate when preferences are non-EU because it is essentially a dynamic version of the independence axiom the non-EU maximizers reject. Machina (1989) proposed to let down consequentialism, for example by conditionning original preference by past uncertainty, that is risk borne, in a consistent manner.

Thus, we see that dynamic inconsistency and Information aversion are closely linked. Shortly, every preference violating Independence axiom displays these types of inconsistency. To overcome these difficulties, authors restrict the class of non-EU preferences by imposing different consistent properties they must satisfy.

Our approach in this paper ${ }^{4}$ is different: instead of starting from agent s preferences to infer agent $\mathrm{s}$ attitude towards information, conversely we start from the attitude towards information (Information Averse, Neutral or Loving) to infer the agent s preferences logically possible. In our knowledge, it is the rst work in which such basic assumptions are proposed. To achieve this goal, we begin by displaying the simplest dynamic version of the Allais paradox, using two strategically equivalent trees, in order to present the different possible attitudes towards information. Then, following a suggestion of Yaari (1985), we characterize this latter attitude in the Choice Functions Theory s framework. We show for instance that an agent who has non-EU preferences can be Information Averse as pointed out by Wakker (1988) but also Information Loving. Therefore, the simple observation of non-EU preferences cannot give us any piece of information about the agent s attitude towards information.

This paper includes six sections. In section 2, we introduce the problem, we present the notion of strategically equivalent decision trees and we de ne attitude towards information. Section 3 is devoted to our main result characterizing attitude towards information in terms of preferences. In section 4, we make an analysis of our results. We show for instance that despite appearance, non-EU agent respects a special dynamic consistency condition we call Cross-levels Dynamic Consistency. Section 5 compares our de nition of attitude towards information with those of Blackwell (1953) and Grant, Kajii and Polack (1998). Finally, section 6 concludes the paper. All proofs are relegated in appendices.

\section{Rationale}

Consider an agent who must choose an action. The problem this agent faces is one of choice under risk if contemplated actions do not have unique consequences. The standard way of describing an action in this case is to write down a list of states-of-nature and to specify what the consequences of the action would be in each state. Thus an action is a rule that associates a unique consequence with every state-of-nature, and it is from among objects of this type that the agent is called upon to choose. In standard decision making under risk, an action is expressed as a lottery and one deals with preferences over lotteries, where in a formal set-up lotteries are modelled as probability distributions over

\footnotetext{
${ }^{4}$ This paper considers the context of decision making under risk, with given probabilities, but it can of course be reformulated in a completely straightforward way for decision making under uncertainty, with sure-thing principle as an analog of independence (Savage (1954)).
} 
consequences called prizes ${ }^{5}$.

An axiom of EU theory in the set of axiomatics by Jensen (1967) is the following known as Independence Axiom : let $r, q$ and $q^{\prime}$ be lotteries belonging to the set of lotteries and let $\lambda \in] 0,1]$ then $q \succ r \Longleftrightarrow \lambda q+(1-\lambda) q^{\prime} \succ \lambda r+(1-\lambda) q^{\prime}$. That is : if lottery $q$ is preferred to lottery $r$ then the compound lottery (a lottery whose prizes are themselves lotteries ${ }^{6}$ ) that leads to lottery $q$ with probability $\lambda$ and to lottery $q^{\prime}$ with probability $1-\lambda$ should be preferred to the compound lottery that leads to lottery $r$ with probability $\lambda$ and to lottery $q^{\prime}$ with probability $1-\lambda$. As shown by Allais (1953) s famous example, this axiom can be violated by agents : take $q^{\prime}=\Phi_{0}$ (the degenerate lottery that gives 0 with probability 1$), r=\widetilde{W}=\left(5 \bar{M}, \frac{10}{11} ; 0 \bar{M}, \frac{1}{10}\right)$ is the lottery that gives 5 millions with probability $\frac{10}{11}$ and 0 million otherwise, $q=\Phi_{1}$ (the degenerate lottery that gives 1 million with probability 1$), \lambda=p=0.11$.

Allais has shown that some people have the following pattern of preferences : $\Phi_{1} \succ \widetilde{W}$ and $p \widetilde{W}+(1-p) \Phi_{0} \succ p \Phi_{1}+(1-p) \Phi_{0}$. In violation of Independence Axiom.

In this paper, we call Non-EU preference such a preference $\succ$ that violates Independence Axiom. There are some theories that allow for such a violation. Their goal is of course to generalize the EU theory and they do it very well. There are however some disturbing things about allowing for Independence Axiom violation.

\subsection{An Agent who violates the Independence Axiom is no longer dynamically consistent}

Let $\succ$ be a preference relation (of an agent) that violates Independence Axiom : $q \succ r$ and $\lambda r+(1-\lambda) q^{\prime} \succ \lambda q+(1-\lambda) q^{\prime}$.

Following Raiffa (1968), decisions under risk in extensive form can be modelled as a decision tree in which boxes $(\square)$ and circles $(\bigcirc)$ denote respectively, decision nodes (where the decision maker (DM) chooses) and chance nodes (where nature chooses). Let us consider the following decision tree that represents the above decision under risk.

\footnotetext{
${ }^{5}$ The kind of prizes that the lotteries yield is immaterial for the present analysis. For instance, prizes may be amounts of money.

${ }^{6}$ Although the successive chance nodes in a compound lottery are resolved sequentially rather than simultaneously, we assume that this process does not require an economically signi cant amount of time and that the Decision Maker has no other economic activities or decisions (e.g. consumption/saving decisions) to undertake in the meantime, so that he has no reason to prefer neither single-stage over compound lotteries nor few-staged over many-staged trees, on ground of impatience or planning bene ts alone. For a discussion of the applicability of decision theory when delays in the resolution of uncertainty are economically signi cant, see Kreps \& Porteus (1978, 1979).
} 


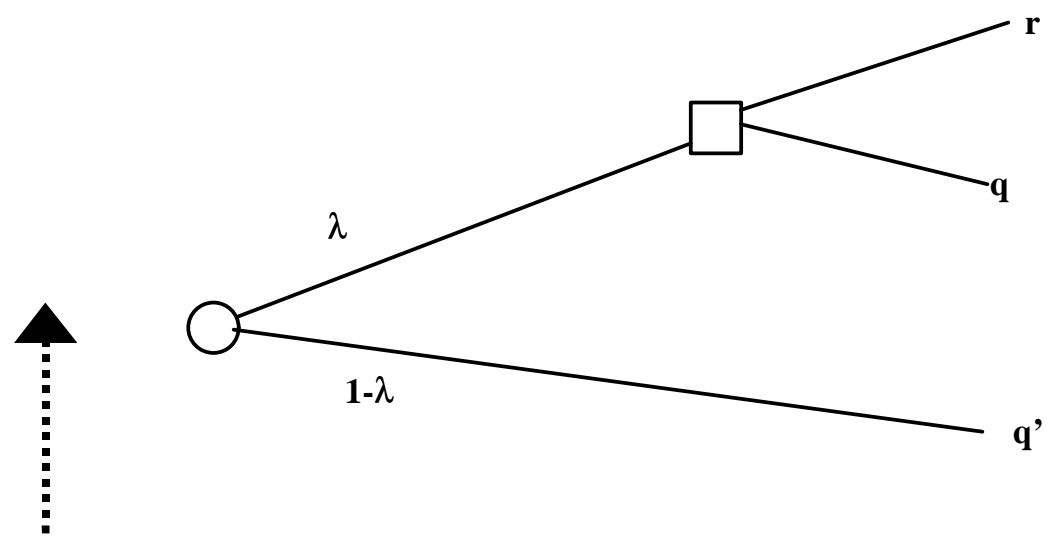

Figure 1

To understand this tree :

1. The DM gets $r$ with probability $\lambda$ and gets $q^{\prime}$ with probability $1-\lambda$, that is $\lambda r+$ $(1-\lambda) q^{\prime}$.

2. The DM gets $q$ with probability $\lambda$ and gets $q^{\prime}$ with probability $1-\lambda$, that is $\lambda q+$ $(1-\lambda) q^{\prime}$.

3. The situation described in this decision tree involves a dynamic setting that is a situation where Nature moves rst and the DM thereafter at least one time.

The DM is not dynamically consistent because ex-ante (at the dotted arrow), he wishes to go DOWN with respect to his preference : $\lambda r+(1-\lambda) q^{\prime} \succ \lambda q+(1-\lambda) q^{\prime}$. But when Nature goes UP, then the DM goes UP with respect to his preference : $q \succ r$.

\subsection{An agent who violates the Independence Axiom is Informa- tion Averse (Wakker, 1988)}

A formal proof of this claim can be found in Schlee (1990). We will give here the intuition of the result. The following two decision trees represent the same decision problem that is a choice between $\lambda q+(1-\lambda) q^{\prime}$ and $\lambda r+(1-\lambda) q^{\prime}$. 



Figure 2

There is however a topological difference between T1 and T2. In T1 the DM chooses rst and Nature thereafter. In T2, Nature chooses rst and DM thereafter.

\section{Examination of T2}

At $\bigcirc$, there is a random device leading to two results : $\mathrm{E} 1$ and $\mathrm{E} 2$ with $\operatorname{Pr}(E 1)=\lambda$ and $\operatorname{Pr}(E 2)=1-\lambda$. If E1 occurs then DM knows that nal outcomes will be either $r$ if he chooses to go UP or $q$ if he chooses to go DOWN. If E2 occurs then DM knows that 
nal outcome will be $q^{\prime}$. Then at T2, DM has an information about the nal outcomes before choosing. Decision tree T2 is said to be more informative ${ }^{7}$ than decision tree T1.

Broadly speaking, the DM will be Information Averse $^{8}$ if he prefers to choose over T1 instead of $\mathrm{T} 2$.

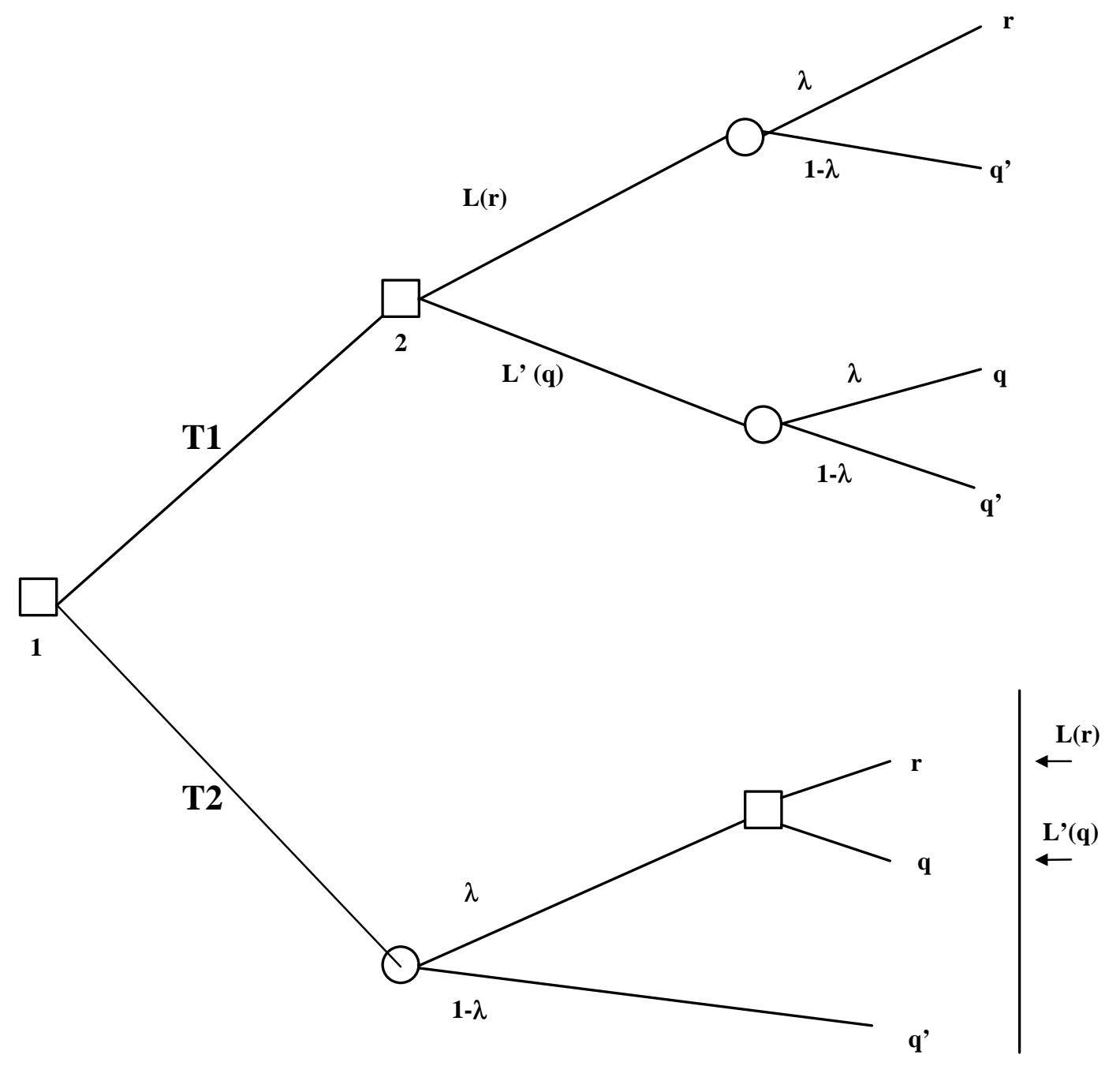

Figure 3

If the DM has to choose over T1 then since he prefers $\lambda r+(1-\lambda) q^{\prime}$ over $\lambda q+(1-\lambda) q^{\prime}$ he will end up with $\lambda r+(1-\lambda) q^{\prime}$. If the DM has to choose over T2 then if Nature moves

\footnotetext{
${ }^{7}$ The formal de nition will be given in section 3 .

${ }^{8}$ The rough de nition will be given in section 3 .
} 
UP then he will end up with $q$ since he prefers $q$ over $r$. But if Nature moves DOWN then he will end up with $q^{\prime}$. Hence if DM has to choose over T2, he will end up with lottery $\left(\lambda, q ; 1-\lambda, q^{\prime}\right)$.

Since the DM prefers $\lambda r+(1-\lambda) q^{\prime}$ then he will prefer to choose over T1 in order to get $\lambda r+(1-\lambda) q^{\prime}$. That is he prefers to choose without information.

Information aversion arises (for instance) in situations where the pay-off function depends to a signal (Schlee (2001), Datta, Mirman and Schlee (2000)). For instance, Lerman et alii (1996) show that $57 \%$ of a group of subjects with a family history of breast/ovarian cancer decline to receive free genetic test results. The main reason is a fear in insurance consequence if the result becomes public.

The result of Wakker (1988) was important in the literature because it has in uenced a research eld that adresses both to dynamic consistency and the role of information in Non-EU theories. To resume, the analysis of Wakker and others, has shown that there exists a link between attitude towards information and preferences displayed by the DM.

The purpose of this paper is to give a complete model-free characterization of attitude towards information in term of preference displayed by the DM. Following a suggestion by Yaari (1985), we use the revealed preference framework. The two mathematical interests of this paper are to adopt a model-free (then universal) approach and to work with decision trees.

\section{Method and De nitions}

\subsection{Preliminary}

Let $\Pi$ be the abstract universal set of nite decision trees. Let us apply the following four operations (see Lavalle (1978), Lavalle \& Fishburn (1987)) over П :

FIRST OPERATION : Delete from $\Pi$ the decision trees obtained from other by Successive Choice Operations (combining or stringing out arcs belonging to the same participant -nature or DM-). For instance if $\Pi$ includes the two trees below then delete the second.

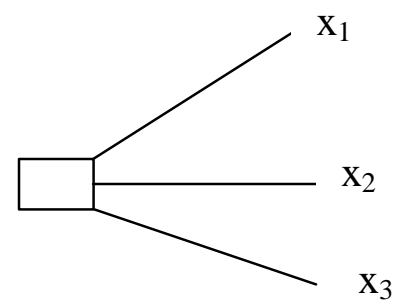

and

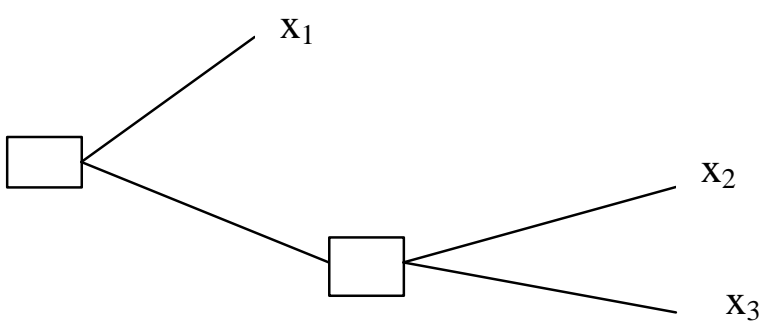

SECOND OPERATION : Delete from $\Pi$ the decision trees obtained from another by Dummy-Move Operations (insertion and/or deletion of dummy moves -nodes from 
which emanates only one arc-). For instance if $\Pi$ includes the two trees below then delete the second.

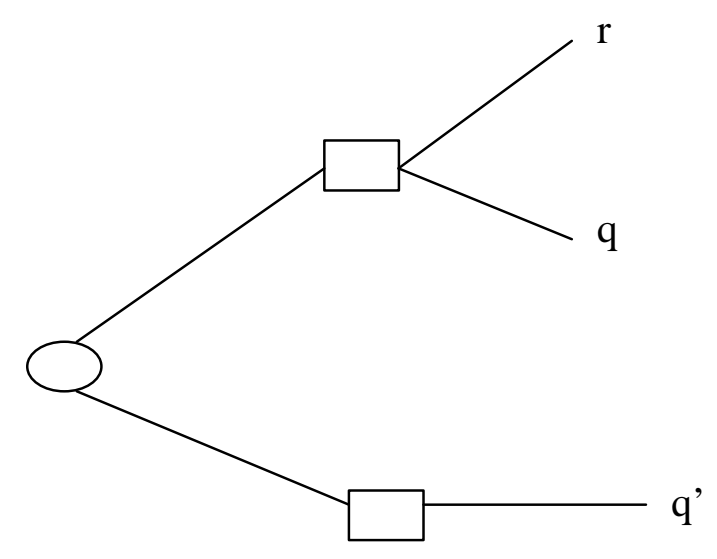

and

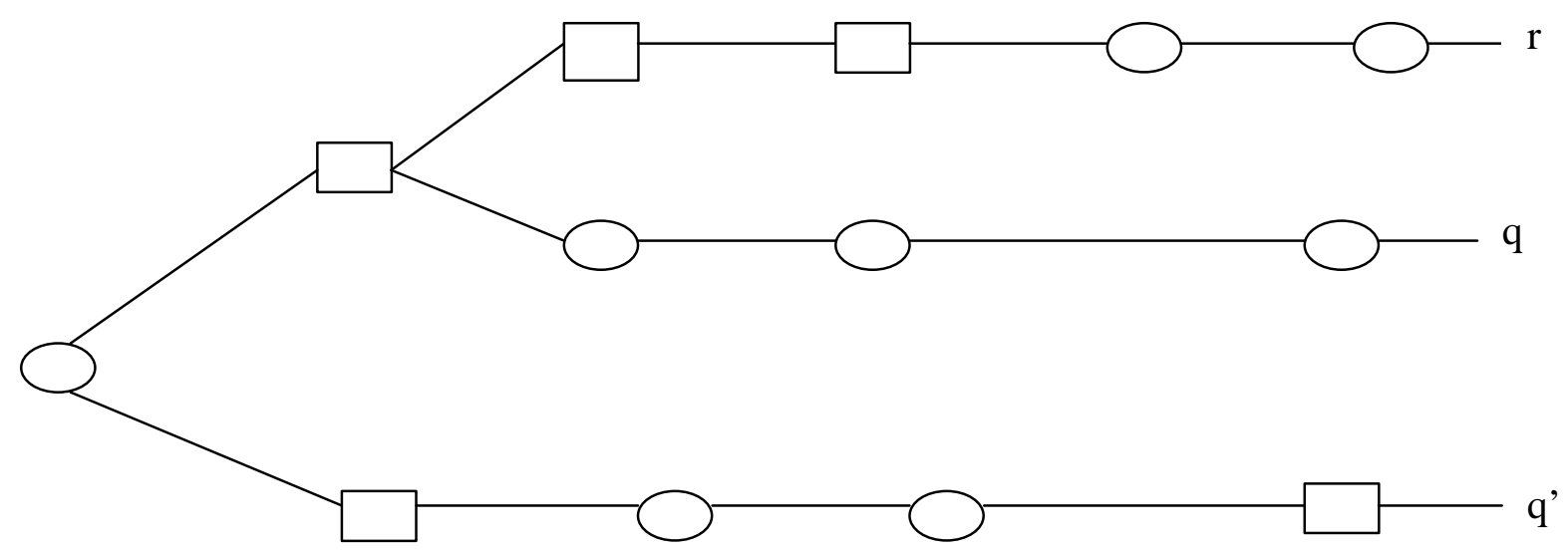

THIRD OPERATION: Delete from $\Pi$ the decision trees obtained from another by a Path Permutations (permutations of the arcs with ensuing subtrees at one or more nodes). For instance if $\Pi$ includes the two trees below then delete the second. 


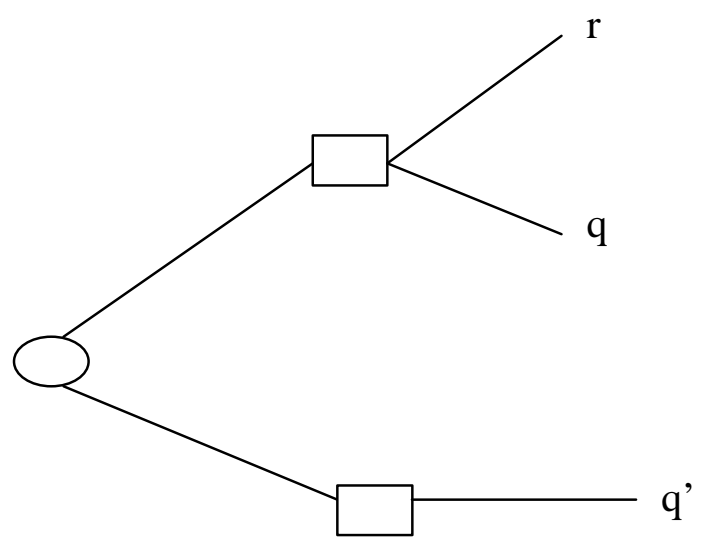

and

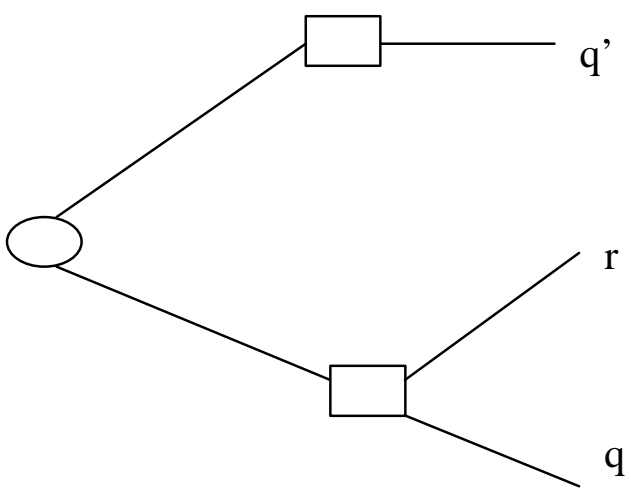

After these rst three operations, we get a set $\Pi^{\prime}$. Let us rename this set by $\Pi$.

FOURTH OPERATION : Apply over $\Pi$ the following equivalence relation denoted SE and de ned by : $\mathrm{T}$ and $\mathrm{T}$ are strategically equivalent, $\mathrm{T}$ SE $\mathrm{T}$, if they have the same opportunity set, that is if they have the same set of endpoints and the same probability distribution over the set of endpoints.

An opportunity set is therefore a set of lotteries.

For instance the above trees T1 and T2 in gure 3 are strategically equivalent.

Loosely speaking, the argument entails that the order of choice of the decision maker and nature may be reversed. Up to operations 1-3, two decision trees (or subtrees) are strategically equivalent from the standpoint of the DM, if they imply the same opportunity set. Therefore, according to the standard analysis, the agent should be indifferent between two strategically equivalent decision trees.

In gure 3, it is obvious that the opportunity set of subtree $1\left(T_{1}\right)$ is $\left\{L(r), L^{\prime}(q)\right\}$. Concerning subtree $2\left(T_{2}\right)$, as argued below, the opportunity set is also $\left\{L(r), L^{\prime}(q)\right\}$. 
Therefore, according to the standard analysis, the individual should be indifferent at the initial choice node $\square 1$ between subtree $T_{1}$ and subtree $T_{2}$.

The argument goes as follows. First, it should be immaterial to the decision maker if he has to choose before (subtree $T_{2}$ ) or after (subtree $T_{1}$ ) the choice of nature concerning whether event E1 or E2 obtains. The argument is that in each case he has the same two compound lotteries or opportunities at his disposal. Second, when he chooses before the nature s choice, the decision maker should act in accordance with the situation in which he chooses after nature.

Nevertheless, the difference between these two situations is relevant, for instance because of the rst situation considerations related to entire probability distributions which are no longer relevant in the second choice situation one.

Let lotteries and mixtures again be as in gure 3. We introduce a new decision problem, a two-stage choice situation as in $\square 1$ of the gure 3. Again, the DM will be faced with the compound lotteries $L(r)$ and $L^{\prime}(q)$. But he must rst make another choice: he must decide whether or not to receive, before choosing between the compound lotteries, the information about whether event E1 or E2 obtains or not. This information is free of charge. So he must choose between a choice situation with no information ( $\square 2$ of the gure 3), where the DM makes his choice between the compound lotteries without knowing what event will actually obtain (for example, the DM must make his choice before nature has decided whether event E1 or E2 will obtain), and a choice situation with information ( $\square 3$ of the gure 3), where he will be informed about whether event E1 or E2 obtains before he has to choose and therefore will have to make his choice after nature has decided whether event E1 or E2 obtains.

For instance, in our decision tree ( gure 3), the DM will be faced with the lotteries $L(r)$ and $L^{\prime}(q)$, and must decide whether or not to receive, before choosing between the two lotteries, the information about whether event E1 or E2 obtains or not.

In gure 3, the DM will not necessarily be indifferent between the two choice situations de ned above. He knows that if he chooses to receive the information, he will end up with the lottery $q^{\prime}$ if event E2 does obtain, and have the choice between the two lotteries $r$ and $q$ otherwise. If he chooses not to receive information, then he will choose between the two compound lotteries $L(r)$ and $L^{\prime}(q)$.

Having done the above partition of $\Pi$ (the abstract universal set of nite decision trees) in classes of strategically equivalent decision trees, we are now able to de ne more precisely the concepts of a tree more informative than another, and of attitude towards information.

\subsection{De nitions}

De nition 1 We call Decision Problem denoted $\mathbb{P}_{i}$, the pair $\left\{\mathbb{O}_{i}, C\right\}$ where $\mathbb{O}_{i}$ is the opportunity set associated with $\mathfrak{C}_{i}$ a class of strategically equivalent trees and $C$ is a choice function. 


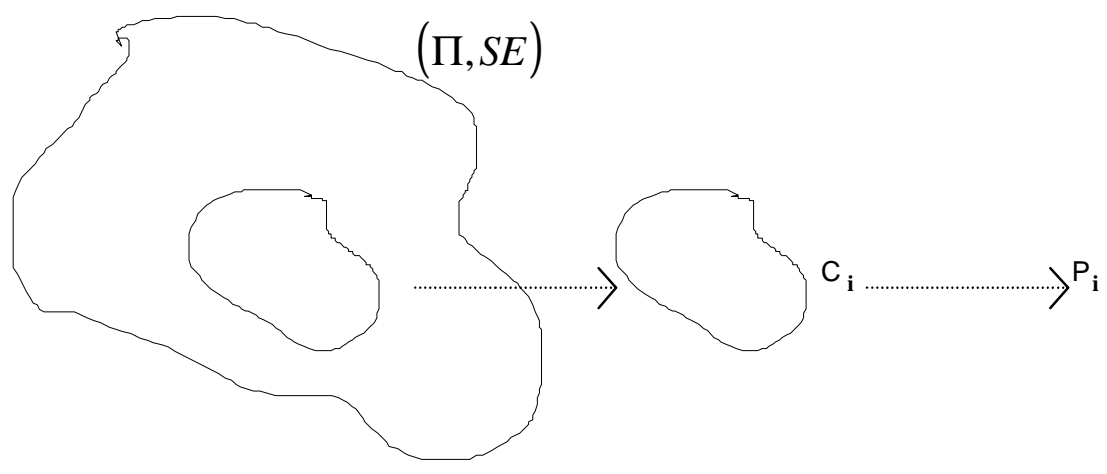

De nition 2 Let $T$ be a decision tree, we call Information Structure of $T$, denoted $I(T)$, the number $I(T)=\underset{d_{i} \in D}{\operatorname{Max}}\left|\operatorname{ch}\left(d_{i}\right)\right|$ where $D$ is the set of decision nodes of $T, d_{i}$ is a decision node $\in D, C H$ is the set of chance nodes of $T, c_{i}$ is a chance node $\in C H, \operatorname{ch}\left(d_{i}\right)=$ $\left\{\begin{array}{c}c h_{i} \in C H: \text { ch } h_{i} \text { is a predecessor } \\ \text { (in the sense of graph theory) of } d_{i}\end{array}\right\}$ and $\left|\operatorname{ch}\left(d_{i}\right)\right|$ is the cardinal of $\operatorname{ch}\left(d_{i}\right)$.

De nition 3 Let $T$ and $T^{\prime}$ belong to a same class $\mathfrak{C}$ of strategically equivalent decision trees. $T$ is more informative than $T$ if $I(T) \geq I\left(T^{\prime}\right)$.

For instance, in gure 4 below, $\mathrm{T}$ is more informative than $\mathrm{T}$ which is more informative than $\mathrm{T}$.

Let us now de ne a preference relation.

De nition $4 A$ preference relation over a set $X$ of objects, is modelled by a binary relation $\succsim$ over $X$ where $\succsim$ is a subset of $X \times X$. $x \succsim y$ means $x$ is preferred to $y$.

De nition 5 Any binary relation $\succsim$ can be parted in a symmetric component called indifference, denoted $\sim$ and de ned by $x \sim y \Longleftrightarrow x \succsim y$ and $y \succsim x$, and in an asymmetric component called strict preference denoted $\succ$ and de ned by $x \succ y \Longleftrightarrow x \succsim y$ and $\operatorname{not}(y \succsim x)$.

De nition $6{ }^{9} A$ binary relation $\succsim$ over $X$ is complete if $\forall x, y \in X, x \succsim y$ or $y \succsim x$.It is transitive if $\forall x, y, z \in X, x \succsim y$ and $y \succsim z$ imply $x \succsim z$.

\footnotetext{
${ }^{9}$ De nition 2 gives us a useful tool to distinguishing a tree more informative than another. Of course, it is possible to set another de nition. For instance : a decision tree $T$ is more informative than a decision tree $T^{\prime}$ if $T^{\prime}=\varphi(T)$ where $\varphi$ is a strategically equivalence preserving transformation such that a decision node (in $T$ ) is permuted with a predecessor chance node.
} 


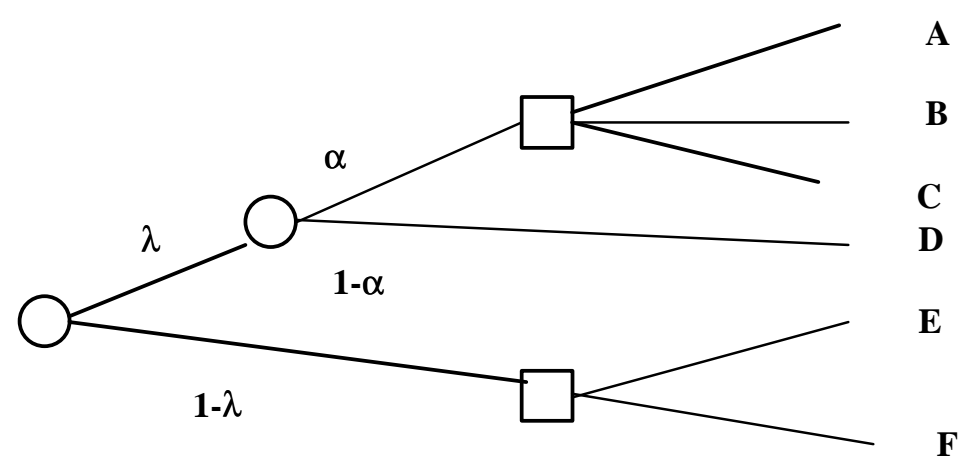

T

$$
\mathbf{I}(\mathbf{T})=\mathbf{2}
$$

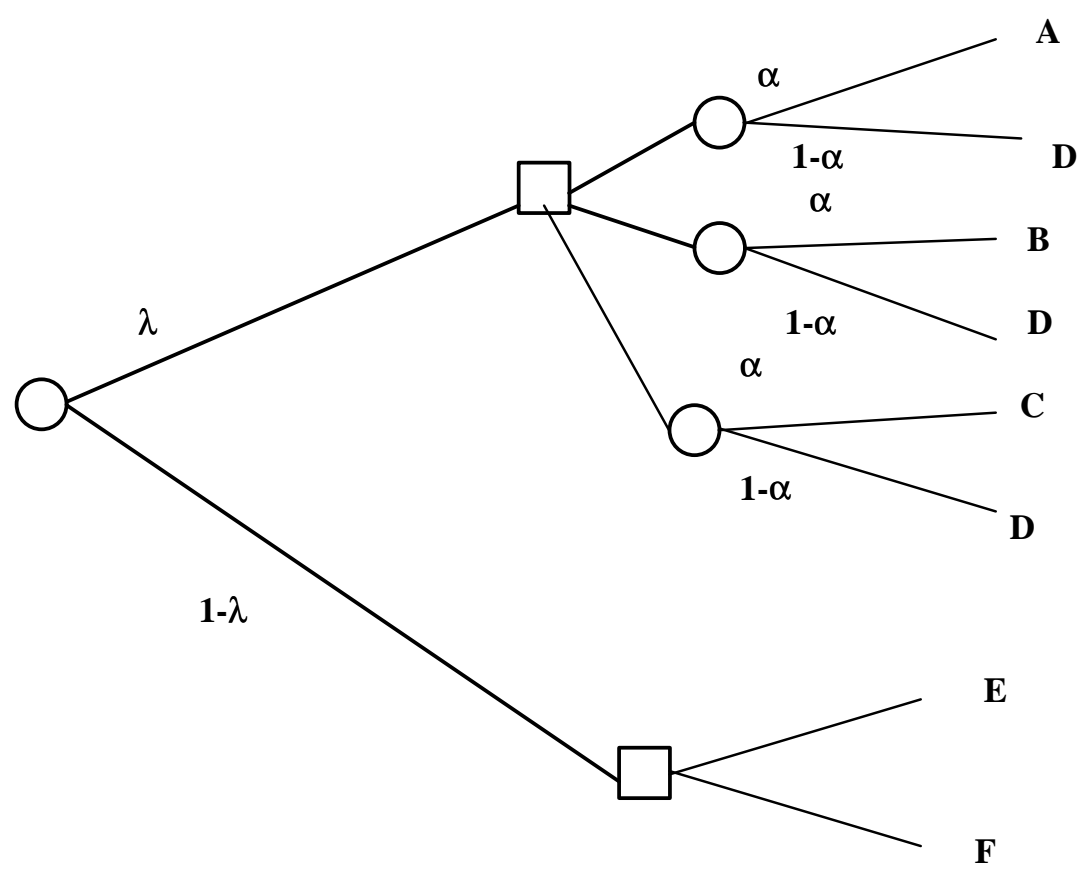

T'

$\mathrm{I}\left(\mathrm{T}^{\prime}\right)=1$ 


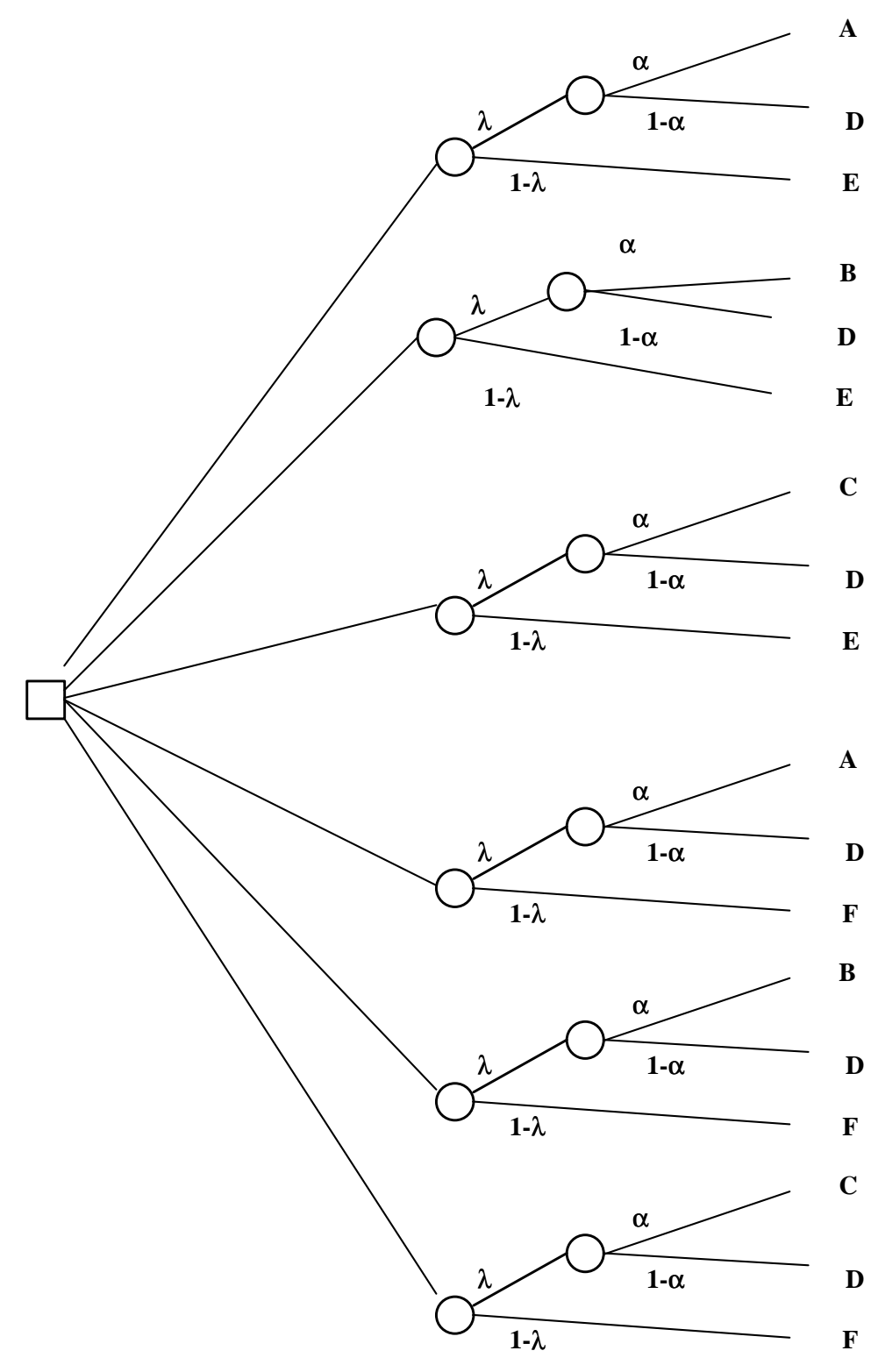

T',

$I\left(T^{\prime \prime}\right)=0$

Figure 4

Let us set the following hypothesis.

H1 The DM admits a preorder (complete and transitive) preference relation $R_{i}$ over $\mathfrak{C}_{i}$. 
There exists therefore a preference relation $R$ over $\cup_{i} \mathfrak{C}_{i}$, which is the disjunction union of the $R_{i}: R={ }_{i} R_{i}$. This preference relation $R$ is of course not complete but it is re exive and transitive. Let us remark that : $R=P_{R}+I_{R}$ with $P_{R}=+P_{R_{i}}$ and $I_{R}={ }_{i} I_{R_{i}}$. With $P_{R_{i}}$ and $I_{R_{i}}$ respectively the asymmetric component and symmetric component of preference relation $R_{i}$.

We will now introduce our de nition of local attitude towards information.

De nition $7 A D M$ is information averse for the decision problem $\mathbb{P}_{i}$ if whatever $T$ and $T^{\prime}$ belonging to $\mathfrak{C}_{i}$ (the class of strategically equivalent decision trees to which $\mathbb{P}_{i}$ is associated with) :

$\cdot I(T)>I\left(T^{\prime}\right) \Longrightarrow T P_{R_{i}} T^{\prime}$

$\cdot I(T)=I\left(T^{\prime}\right) \Longrightarrow T I_{R_{i}} T^{\prime}$

where $P_{R_{i}}$ is the asymmetric component of preference relation $R_{i}$ over $\mathfrak{C}_{i}$ and $I_{R_{i}}$ is the symmetric component of preference relation $R_{i}$.

We have similar de nition concerning information loving for the decision problem $\mathbb{P}_{i}$.

De nition $8 A D M$ is information neutral for the decision problem $\mathbb{P}_{i}$ if whatever $T$ and $T^{\prime}$ belonging to $\mathfrak{C}_{i}$ (the class of strategically equivalent decision trees to which $\mathbb{P}_{i}$ is associated with), we have $T I_{R_{i}} T^{\prime}$ where $I_{R_{i}}$ is the symmetric component of preference relation $R_{i}$ over $\mathfrak{C}_{i}$.

De nition 9 A DM is information averse if he is information averse for any decision problem $\mathbb{P}_{i}$.

We have similar de nitions concerning information loving and information neutrality.

Remark 1 Of course, global attitude towards information implies local attitude towards information.

However Lemma 5 (appendix D) shows the equivalence between attitude towards information and attitude towards information for problem :

$$
\begin{gathered}
\mathbb{P}=\left\{\mathbb{O}=\left\{\lambda r+(1-\lambda) q^{\prime}, \lambda q+(1-\lambda) q^{\prime}\right\} ; C\right\} \\
\forall \lambda \in] 0,1\left[, \quad r, q \text { and } q^{\prime}\right. \text { are lotteries. }
\end{gathered}
$$

This result allows us to deal with the decision problem :

$$
\mathbb{P}=\left\{\mathbb{O}=\left\{\lambda r+(1-\lambda) q^{\prime}, \lambda q+(1-\lambda) q^{\prime}\right\} ; C\right\}
$$

as a generic one, and with $\mathfrak{C}$ its associated class of strategically equivalent decision trees. We consider, without loss of generality (see part 2 of the proof of Lemma 5 in Appendix D), that this set $\mathfrak{C}$ includes only decision trees T1 and T2 of gure 3. Our below theorem and propositions are therefore general.

We will complete this section with the following two hypotheses. 
H2 Let $\mathbb{P}_{i}$ be a decision problem and $\mathfrak{C}_{i}$ its associated class of strategically equivalent decision trees. Whatever $T$ and $T^{\prime}$ belonging to $\mathfrak{C}_{i}, I(T)=I\left(T^{\prime}\right) \Longrightarrow T I_{R_{i}} T^{\prime}$.

Hypothesis $\mathrm{H} 2$ says that if two strategically equivalent decision trees have the same information structure then the DM is indifferent between both. This hypothesis allows us to focus over the strategically equivalent decision trees having different information structures. Assuming H2, we can rewrite the de nitions of attitudes towards information by neglecting the condition : $\forall T, T^{\prime} \in \mathfrak{C}_{i}, I(T)=I\left(T^{\prime}\right) \Longrightarrow T I_{R_{i}} T^{\prime}$.

H3 Let $\mathbb{P}_{i}$ be a decision problem and $\mathfrak{C}_{i}$ its associated class of strategically equivalent decision trees. Whatever $T$ and $T^{\prime}$ belonging to $\mathfrak{C}_{i}, T \neq T^{\prime}, T P_{R_{i}} T^{\prime}$ [resp. $\left.T I_{R_{i}} T^{\prime}\right] \Longrightarrow \forall Z$ a decision tree, $\forall \lambda \in[0,1]$,



$\mathrm{P}_{\mathrm{R}}\left[\right.$ resp. $\left.\mathrm{I}_{\mathrm{R}}\right]$

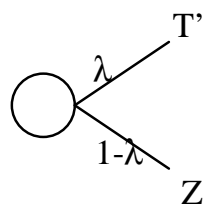

$\mathrm{H} 3$ is an independence axiom over strategically equivalent decision trees but it does not imply the standard independence axiom.

\section{Characterizing attitudes towards information in terms of preferences}

Since we will use the concept of choice function, let us give some basic notions in choice function theory.

Let $X$ be a set of objects. A set of subsets of $X$, denoted $F$, is called domain of choice and the elements of $F$ are called choice sets.

A choice function is a function $C$ de ned from $F$ to $P(X)$ the power set, which associates $S$ with $C(S)$, with the condition $C(S) \subseteq S$.

$C(S)$ is the set of chosen elements over $S$. Moreover, let us assume that :

H4 $C(S)$ is non-empty for any $S$ belonging to $F$, that is $C$ is decisive over $F$. 
De nition 10 (Richter (1971)). A choice function $C$ is said to be Rational if there exists a binary relation $\succsim$ over $X$, rationalizing it, that is, such that for any $S \in F$, $C(S)=\{x \in S: x \succsim y, \forall y \in S\}$.

Thus, a choice function $C$ is rational if the Decision Maker chooses the elements which are optimal with respect to his preference relation.

From a choice function $C$, one can de ne the following binary relation over $X$ called Revealed Preference denoted $\mu$. An object $x$ is revealed preferred to an object $y$ if there exists a choice set over which $x$ is chosen while $y$ could have been. Formally,

De nition $11 \forall x, y \in X, x \mu y \Longleftrightarrow \exists S \in F$ such that $x \in C(S)$ and $y \in S$.

Richter (1971) gives an interesting characterization of rational choices by showing that any choice function is rational if and only if it is rationalizable by $\mu$, the revealed preference relation. Therefore, from a rationality standpoint, any binary relation rationalizing a choice function is equivalent to $\mu$. The revealed preference s concept is hence central in the theory of choice functions.

The above Richter de nition has a dual equivalent (in duality) de nition.

De nition 12 (Kim and Richter (1986)). A choice function $C$ is said to be Rational if there exists a binary relation $\succ$ over $X$, such that for any $S \in F, C(S)=$ $\{x \in S: \operatorname{not}(y \succ x), \forall y \in S\}$.

Let us now take gure 3 where the DM faces the following problem of decision: At the decision node $\square 1$, he has the choice between having the choice at the decision node $\square$ 2 between compound lotteries $L(r)$ and $L^{\prime}(q)$, and the following lottery: with probability $\lambda$, he will have the choice (at the decision node $\square$ 3) between $r$ and $q$ and the lottery $q^{\prime}$ otherwise.

Formally, this decision problem can be written $C_{1}\left\{C_{2}\left\{L(r), L^{\prime}(q)\right\} ;\left[\lambda, C_{3}\{r, q\} ; 1-\lambda, q^{\prime}\right]\right\}$ where $C_{i}$ is the DM s choice function at the decision node $i=1,2,3$.

It is important to stress out that the choice functions $C_{i}$ are a priori pairwise different. The set of elements the DM faces at decision node $\square 1$ is the following set of decision trees $X_{1}=\left\{T_{1}, T_{2}, L(r), L^{\prime}(q)\right\}$ where the compound lotteries $L(r)$ and $L^{\prime}(q)$ ought to be seen here as degenerate decision trees. Indeed it is straightforward to see that the DM faces at decision node $\square 1$, subtrees $T_{1}$ and $T_{2}$ but at this decision node $\square 1$, he is also aware about the existence of lotteries $L(r)$ and $L^{\prime}(q)$ (recall that $T 1$ and $T 2$ are strategically equivalent). The only technical consequence of assuming $L(r)$ and $L^{\prime}(q)$ to belong to the agent s set of decision trees at decision node $\square 1$, is that it allows to well de ne the choice function $C_{1}$ at decision node $\square$. Nevertheless this implicitely implies a perfect foresight at decision node $\square 1$.

The set of elements, the DM faced at decision nodes $\square 2$ and $\square 3$ are respectively $X_{2}=\left\{L(r), L^{\prime}(q)\right\}$ and $X_{3}=\{r, q\}$.

Since we assume the choice functions $C_{i}$ to be a priori different then the (revealed) preferences associated with these choice functions are a priori different.

Let us recall that in traditional analysis, the individual has a preference relation $\succsim$ over the set $\left\{L(r), L^{\prime}(q), r, q\right\}=X_{2} \cup X_{3}$. We can therefore always link our analysis to the 
traditional one by assuming that the relations $\underset{2}{\succsim}$ and $\underset{3}{\succsim}$, respectively preferences relations over $X_{2}$ and $X_{3}$, are subrelations of $\succsim$. Then we have the main result of this paper:

Theorem 1 For strategically equivalent subtrees, an individual who exhibits non-EU preferences $\left(L(r) \succ L^{\prime}(q)\right.$ and $q \succ r$ or $L^{\prime}(q) \succ L(r)$ and $r \succ q$, where $\succsim$ is a preference relation over $\left.\left\{L(r), L^{\prime}(q), r, q\right\}\right)$ can be either

a) Information Averse,

or

b) Information Loving,

or

c) Information Neutral.

Proof. See Appendices A, B, C and D.

Let us comment this result.

Let us recall that the purpose of this paper is to characterize in terms of preferences over the lotteries, the agent s attitude towards information. Theorem 1 enlights that the simple observation of Non-EU preferences cannot give us any information about the agent $s$ attitude towards information.

Since each pattern of preferences between $L(r)$ and $L^{\prime}(q)$ and between $r$ and $q$ (at the different decision nodes) is associated with an attitude towards information, thus an immediate implication of Theorem 1 is that the simple observation of preferences at decision nodes $\square 2$ and $\square 3$ is not sufficient to inform us about the consistency of the choices displayed by the agent. That is, the traditional level of abstraction is not sufficient and at such a level, one cannot impose a dynamic consistency between the choice exhibited at decision nodes $\square 2$ and $\square$. The best level of abstraction (if we want to catch the agent s attitude towards information) must include the preference $\underset{1}{\succsim}$ between $L(r)$ and $L^{\prime}(q)$ at decision node $\square 1$. Let us call Prime Preference Relation, the preference relation $\underset{1}{\succsim}$ at decision node $\square 1$. In that case, the true dynamic consistency criterion should be the following:

\section{Axiom 1 (Cross-levels Dynamic Consistency Axiom)}

For strategically equivalent subtrees $T_{1}$ and $T_{2}$, the agent $s$ preference at decision node 1 between $L(r)$ and $L^{\prime}(q)$ must be consistent with the one expressed, at decision node $\square$ if the agent is Information Averse, at decision node $\square 3$ if the agent is Information Loving and with those expressed at decision node $\square 2$ and $\square 3$ if the agent is Information Neutral.

It is easy to see that :

Remark 2 The following assertions are true. 
1. An Information Averse Decision Maker respects the Cross-levels Dynamic Consistency Axiom.

\section{An Information Loving Decision Maker respects the Cross-levels Dynamic Con- sistency Axiom.}

Comments of Remark2. It says that despite appearance, Information Averse or Information Loving agent is dynamically consistent in the sense that his prime preference between $L(r)$ and $L^{\prime}(q)$ at decision node $\square 1$ is in accordance with his preferences at decision node $\square 2$ where he effectively chooses if he is Information Averse, and with his preferences at decision node $\square 3$ where he effectively chooses if he is Information Loving. Of course, there is no reason to request preferences at decision nodes $\square 2$ and $\square 3$ to be dynamically consistent, and in fact they do not.

Let us take for instance an Information Averse DM with the following preference (Type 1 in the Lemma 1, Appendix A): $L(r) \succ_{1} L^{\prime}(q), L(r) \succ_{2} L^{\prime}(q)$ and $q \succ_{3} r$.

Thus, he strictly prefers $L(r)$ to $L^{\prime}(q)$ at decision node $\square 1$. This preference is his prime preference $\underset{1}{\succsim}$ between $L(r)$ and $L^{\prime}(q)$. Since he is Information Averse, then the DM moves to subtree $T_{1}$ and when he has to choose (at decision node $\square 2$ ) between $L(r)$ and $L^{\prime}(q)$, he chooses $L(r)$ in accordance with his prime preference. However, his choice at decision node $\square 3$ between $r$ and $q$ is not in accordance with his prime preference since he chooses $q$ (which -the choice- is strategically equivalent to $L^{\prime}(q)$ ) and not $r$ (which is strategically equivalent to $L(r))$. The question is why?

We think that the reason is the following: the Decision Maker is Information Averse but at decision node $\square$, he has to choose with information and this has an in uence on his behavior. Through what mechanism remains an open question for us. However, we suspect that information in uences choices through an in uence over the degree of pessimism/optimism and the attitude towards risk (or uncertainty).

Likewise, let us take an Information Loving DM with the following preference (Type 3 in the Lemma 2, Appendix B): $L(r) \succ_{1} L^{\prime}(q), L^{\prime}(q) \succ_{2} L(r)$, and $r \succ_{3} q$.

Therefore, he strictly prefers (prime preference $\underset{1}{\succsim)} L(r)$ to $L^{\prime}(q)$ at decision node

1. Since he is Information Loving, then the DM moves to subtree $T_{2}$ and when he has to choose (at decision node $\square 3$ ) between $q$ (which is strategically equivalent to $L^{\prime}(q)$ ) and $r$ (which is strategically equivalent to $L(r)$ ), he chooses $r$ in accordance with his prime preference. However, his choice at decision node $\square 2$ between $L(r)$ and $L^{\prime}(q)$ is not in accordance with his prime preference since he chooses $L^{\prime}(q)$ and not $L(r)$. The reason is the same as above: the DM is Information Loving but at decision node $\square 2$, he has to choose without information and this has an in uence on his behavior.

Generally speaking, it seems that being Information Averse (respectively Information Loving) and choosing with (respectively without) information, has an in uence on the agent s choices. Nevertheless, the displayed preference $\succsim$ over $\left\{L(r), L^{\prime}(q), r, q\right\}$ is wellde ned from a set-theory standpoint and its restriction to $\left\{L(r), L^{\prime}(q)\right\}$ is dynamically 
consistent with the restriction of prime preference $\underset{1}{\succsim}$ over $\left\{L(r), L^{\prime}(q)\right\}$ if the DM is Information Averse and the restriction of $\succsim$ to $\{r, q\}$ is dynamically consistent with the restriction of prime preference $\underset{1}{\succsim}$ over $\left\{L(r), L^{\prime}(q)\right\}$ if the DM is Information Loving.

What s about an Information Neutral DM?

Proposition 1 The following two conditions are true.

1. An Information Neutral Decision Maker (DM) does not necessarily respect the Crosslevels Dynamic Consistency Axiom.

2. A Decision Maker is Information Neutral and respects the Cross-levels Dynamic Consistency Axiom if and only if he exhibits preferences of type $H$ (that is, $L(r) \sim$ $L(r), L^{\prime}(q) \underset{1}{\sim} L^{\prime}(q), L(r) \underset{1}{\sim} L^{\prime}(q), L(r) \underset{2}{\sim} L^{\prime}(q)$ and $\underset{3}{\sim} q$. See Appendix $\left.C\right), C$ or $D$ where

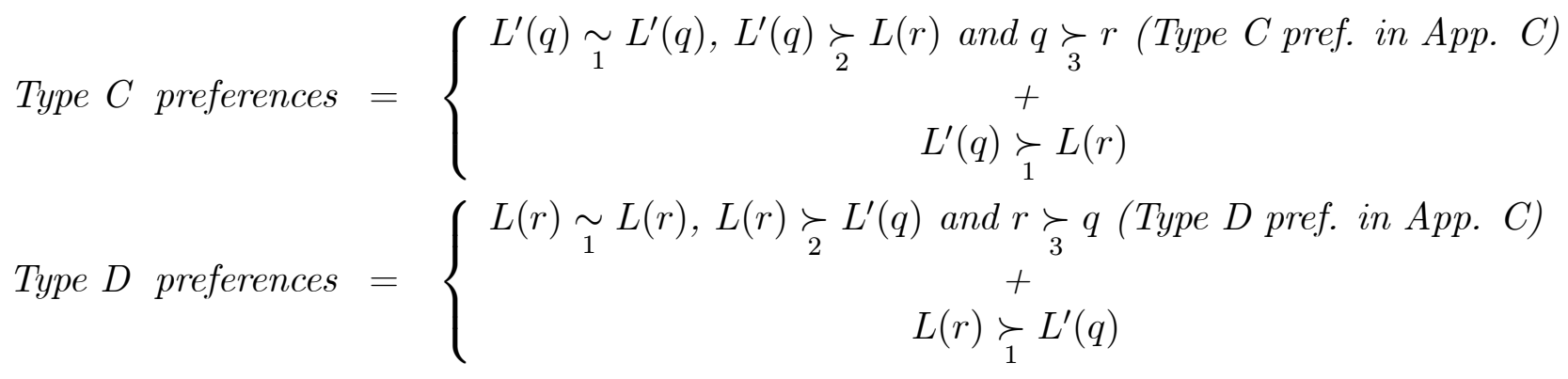

\section{Proof.}

1. Among the nine types of preferences speci ed in Lemma 3 (see Appendix C), only the types $\mathrm{C}, \mathrm{D}$ and $\mathrm{H}$ do not violate Axiom 1.

2. ( $\Longrightarrow$ ) According to condition 1 of the current Proposition, only the $\mathrm{C}, \mathrm{D}$ and $\mathrm{H}$ types of preferences do not violate Axiom 1 . In types $C$ and $D$, the preference $\underset{1}{\succsim}$ at decision node $\square 1$ between $L(r)$ and $L^{\prime}(q)$ is not speci ed but the respects of Axiom 1 requires that $L^{\prime}(q) \succ_{1} L(r)$ in type $\mathrm{C}$ and that $L(r) \succ_{1} L^{\prime}(q)$ in type $\mathrm{D}$.

$(\Longleftarrow)$ If the DM has preferences of type C , D or $\mathrm{H}$ then he is Information Neutral according to Lemma 3. He obviously respects Axiom 1.

Corollary 1 Let us restrict ourselves to the class of Cross-levels Dynamic Consistent Preferences, then the following two conditions are equivalent.

1. The agent is Information Neutral.

2. The agent s preferences are of EU-type. 
Proof. Immediate.

Comments. An agent who is Information Averse or Information Loving necessarily displays non-EU preferences (theorem 1). However, according to Remark 2, such an agent is dynamically consistent in the sense of Axiom 1. Paradoxically, condition 1 of Proposition 1 says that an Information Neutral agent is not necessarily dynamically consistent in the sense of Axiom 1. Condition 2 of the same Proposition speci es the preferences that are Cross-levels dynamically consistent in the case of an Information Neutral agent. And, according to corollary 1, these preferences coincide with those of EUtype. Thus, if we want preference to be dynamically consistent with respect to Axiom 1, then having EU-type preference is equivalent to be Information Neutral (and having non-EU-type preference is equivalent to be Information Averse or Information Loving).

Let us now talk about the traditional justi cation that non-EU preferences are not dynamically consistent. This justi cation says that if two trees are strategically equivalent then the DM s choice should be the same over the two trees while in non-EU preference, that is clearly not the case.

Let us therefore set the following axiom.

Axiom 2 (Strategically Equivalent-Same Choice Axiom).

The Decision Maker s choice should be the same over two strategically equivalent trees.

We obtain the following proposition:

Proposition 2 For strategically equivalent subtrees $T 1$ and $T 2$, the following two conditions are equivalent.

1. The Strategically Equivalent-Same Choice Axiom is ful lled.

2. The preferences are of type $C\left(L^{\prime}(q) \sim L^{\prime}(q), L^{\prime}(q) \succ_{2} L(r), q \succ_{3} r\right)$, type $D$ $\left(L(r) \underset{1}{\sim} L(r), L(r) \succ_{2} L^{\prime}(q), r \succ_{3} q\right)$ or type $H\left(L(r) \underset{1}{\sim} L(r), L^{\prime}(q) \underset{1}{\sim} L^{\prime}(q)\right.$, $\left.L(r) \underset{1}{\sim} L^{\prime}(q), L(r) \underset{2}{\sim} L^{\prime}(q), r \underset{3}{\sim} q\right)$.

Proof.

- $(1) \Longrightarrow(2)$

The DM is either Information Neutral, Information Averse or Information Loving. However, in the cases he is Information Averse or Information Loving, he does not respect the Strategically Equivalent-Same Choice Axiom. And in the case he is Information Neutral, only the C, D and H-types ful ll Axiom 2.

- $(2) \Longrightarrow(1)$ is immediate. 
Corollary 2 For strategically equivalent subtrees $T 1$ and T2, the respect of the Strategically Equivalent-Same Choice Axiom implies that the DM is Information Neutral (and has EU-type preferences).

Proof. Immediate.

Comments. According to Proposition 2, if we want both that the DM considers subtrees 1 and 2 as strategically equivalent and respects the Strategically EquivalentSame Choice Axiom, then we are obliged to restrict ourselves to the class of C, D and H-types preferences, hence (Corollary 2) to suppose that the DM is Information Neutral.

Let us nish with the following remark. Despite the concept of strategical equivalence is objective, the DM may subjectively consider subtrees 1 and 2 as not strategically equivalent (likewise, the DM may subjectively consider strategically equivalent two decision trees which are not objectively strategically equivalent), that is, up to conditions 1-3 of de nition 1, the DM considers that subtrees 1 and 2 do not have the same opportunity set. For instance when the DM respects the axiom called Forgone-Event Independence Axiom by Wakker (1999) and Consequentialism by Machina (1989), that is independence of past counterfactual events, then he clearly subjectively considers that $T_{1}$ and $T_{2}$ are not strategically equivalent. Indeed $T_{2}$ becomes the subtree starting from decision node $\square 3$ and the opportunity set of this subtree is $\{r, q\}$ while the one of $T_{1}$ remains $\left\{L(r), L^{\prime}(q)\right\}$.

\section{Related Litterature}

Following Blackwell s (1953) theorem according to which all agents (at least weakly) prefer more information to less, most of the litterature on individual choice for information (e.g. Wakker (1988) or Schlee (1990)) chooses to focus on instrumental preference for information, i.e. a decision maker likes information only because it lets him design better strategies. Thus, if he does not or cannot condition his actions on what he learns, information is of no value for him. Introspection suggests however that we sometimes intrinsically prefer more information to less, even in absence of any instrumental purpose. Moreover, Blackwell s original setup is quite restrictive in the way it models attitudes towards both risk and information. For example, if Blackwell s agent always prefers the complete elimination of a risk (the mean of the original distribution), then he always prefers any partial removal of the risk (any mean-preserving contraction). This is a consequence of the expected utility hypothesis. At last, the rare attempts (the seminal works of Wakker (1988), Machina (1989) and Schlee (1990)) to extend standard individual choices models of preference for information in a non-expected utility framework favor preference for information as an axiom of individual choice.

In our knowledge, Grant, Kajii \& Pollak (1998) is the only paper explorating the connections between risk and information by focusing on intrinsic preferences for information. Introspection suggests that attitude towards risk and attitude towards information are closely related. They show that the way in which they model an agent s attitude towards risk has implication for his attitude towards information and vice versa. For example, if 
you wish to maintain Blackwell s result that an agent always prefers more information to less, you have to restrict the overall shape of the agent s preferences over risky prospects. Moreover, they show that intrinsic preference for information is equivalent to a simple substitution property (Single-Action Information Loving (SAIL) property) of preferences over two-stage lotteries. Since this substitution property is de ned directly on preferences, not on any particular functional representation, it is applicable to all non-expected utility models. SAIL property is related to attitudes towards risk in two quite different ways. First, preference for information restricts how an agent $\mathrm{s}$ attitude towards risk in lotteries that resolve early compare to his attitude towards risk in lotteries that resolve late. Second, SAIL property is analogous to risk loving with respect to the distribution of posteriors. Intuitively, information causes posteriors to be more widely dispersed. Loosely speaking, this analogy allows them to translate known results about attitudes towards risk into new results about attitude towards information.

We use also a concept of Intrinsic Preferences for Information but over Decision trees while Grant, Kajii \& Pollak (1998) deal with lotteries. For instance, Decision tree $T_{2}$ can be seen as a linear bifurcation of $T_{1}$.

\section{Conclusion}

The main nding of this paper is that the current level of abstraction used by non-EU theories is not sufficient to catch all the aspects of the agents behaviors. This level only deals with the agents preferences over a set of lotteries while the best level of abstraction should also include the agents preference over the set of decision trees that can be derived from the set of lotteries. This latter preference (we call prime preference) is different in its nature from the one over the set of lotteries since prime preference denotes an attitude towards information and the preference over the set of lotteries, an attitude towards risk (or uncertainty). Such a modelling can for instance be found in Grant, Kajii and Polak (1998). Of course, attitude towards information has an in uence over preferences displayed over the set of lotteries. We show that agents in non-EU theories respect in fact a (Cross-levels) Dynamic Consistency Condition.The results are however valid for strategically equivalent decision trees. 


\section{A Proof of Theorem 1 a)}

Lemma 1 Let a DM have choice functions $C_{i}, i=1,2,3$, when choosing over the sets $X_{1}=\left\{T_{1}, T_{2}, L(r), L^{\prime}(q)\right\}, X_{2}=\left\{L(r), L^{\prime}(q)\right\}$ and $X_{3}=\{r, q\}$. For strategically equivalent subtrees $\mathbf{T}_{1}$ and $\mathbf{T}_{2}$, the following two conditions are equivalent.

1. The DM is Information Averse.

2. The DM s preferences at nodes $\square 1, \square 2$ and $\square 3$ denoted respectively $\underset{1}{\succsim}$, $\underset{2}{\succsim}$ and $\underset{3}{\succsim}$ are either of

- Type 1, that is

$1.1 L(r) \succ L^{\prime}(q)$

$1.2 L(r) \succ_{2} L^{\prime}(q)$

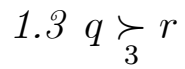

or of

- Type 2 , that is

2.1 $L^{\prime}(q) \succ L(r)$

2.2 $L^{\prime}(q) \succ_{2} L(r)$

$2.3 r \succ_{3} q$

Proof.

- $(1) \Longrightarrow(2)$

If the DM is Information Averse then he chooses to choose directly between compound lotteries $L(r)$ and $L^{\prime}(q)$, that is

$$
C_{1}\left\{C_{2}\left\{L(r), L^{\prime}(q)\right\} ;\left[\lambda, C_{3}\{r, q\} ; 1-\lambda, q^{\prime}\right]\right\}=C_{2}\left\{L(r), L^{\prime}(q)\right\}
$$

Let $\underset{1}{\succsim}$ be the DMs (revealed) preference relation de ned over the set $X_{1}$. The DM strictly prefers $\left(\succ_{1}\right)$ at node $\square 1, C_{2}\left\{L(r), L^{\prime}(q)\right\}$ to $\left[\lambda, C_{3}\{r, q\} ; 1-\lambda, q^{\prime}\right]$, that is:

- At node $\square 1, C_{2}\left\{L(r), L^{\prime}(q)\right\} \succ_{1}\left[\lambda, C_{3}\{r, q\} ; 1-\lambda, q^{\prime}\right]$

- $\underline{\text { At node } \square 2}, C_{2}\left\{L(r), L^{\prime}(q)\right\}$ gives three possibilities of choice: choosing $L(r)$ alone, choosing $L^{\prime}(q)$ alone, or choosing both.

- At node $\square 3, C_{3}\{r, q\}$ gives also three possibilities: choosing $r$ alone, choosing $q$ alone, or choosing both.

That leads to 9 cases. Let us represent these cases by the following graph: 


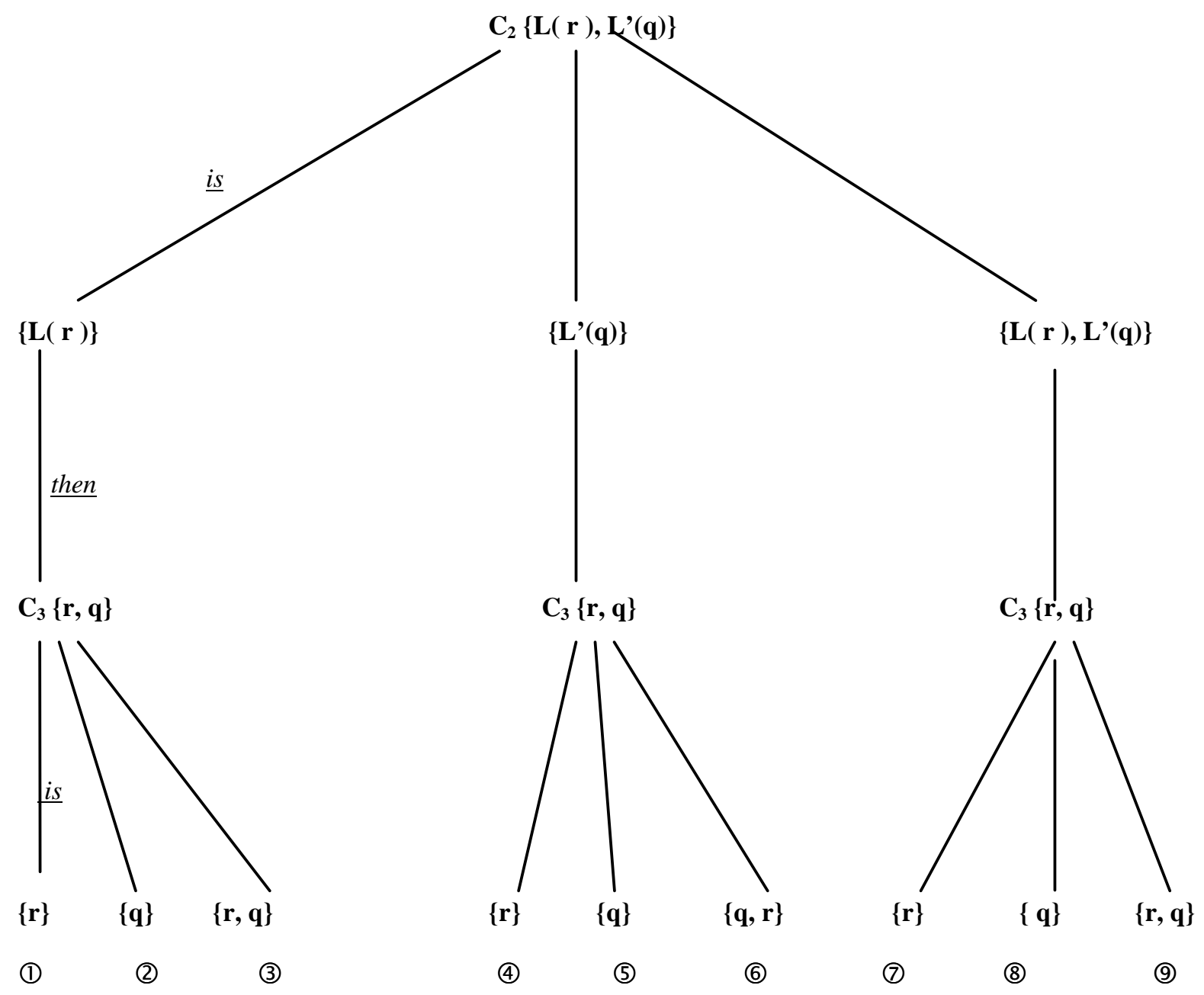

Let us consider cases 2 and 4 .

* Case 2:

Since $C_{2}\left\{L(r), L^{\prime}(q)\right\}=\{L(r)\}$ then at node $\square 2, L(r) \succ_{2} L^{\prime}(q)$. Since $C_{3}\{r, q\}=q$ then at node $\square 3, q \succ_{3}$. Therefore $C_{2}\left\{L(r), L^{\prime}(q)\right\} \succ_{1}\left[\lambda, C_{3}\{r, q\} ; 1-\lambda, q^{\prime}\right]$ is equivalent to $L(r) \succ_{1}\left[\lambda, q ; 1-\lambda, q^{\prime}\right]$, that is $L(r) \succ_{1} L^{\prime}(q)$.

* Case 4:

Since $C_{2}\left\{L(r), L^{\prime}(q)\right\}=\left\{L^{\prime}(q)\right\}$ then at node $\square 2, L^{\prime}(q) \succ_{2} L(r)$. Since $C_{3}\{r, q\}=r$ then at node $\square 3, r \succ_{3} q$. Therefore $C_{2}\left\{L(r), L^{\prime}(q)\right\} \succ_{1}\left[\lambda, C_{3}\{r, q\} ; 1-\lambda, q^{\prime}\right]$ is equivalent to $L(r) \succ_{1}\left[\lambda, r ; 1-\lambda, q^{\prime}\right]$, that is $L^{\prime}(q) \succ_{1} L(r)$. 
The other cases $1,3,5,6,7,8$ and 9 are logically impossible. Let us make the proof for case 1 for example.

Since $C_{2}\left\{L(r), L^{\prime}(q)\right\}=\{L(r)\}$ then at node $\square 2, L(r) \succ L^{\prime}(q)$. Since $C_{3}\{r, q\}=r$ then at node $\square 3, r \succ_{3} q$. Therefore $C_{2}\left\{L(r), L^{\prime}(q)\right\} \succ\left[\lambda, C_{3}\{r, q\} ; 1-\lambda, q^{\prime}\right]$ is equivalent to $L(r) \succ_{1}\left[\lambda, r ; 1-\lambda, q^{\prime}\right]$, that is $L(r) \succ_{1} L(r)$, which is logically impossible by de nition of strict preference as asymmetric (thus irre exive) component of preference relation.

- $(2) \Longrightarrow(1)$

Let a DM exhibiting a pro le of preference relations of type 1 , then since $q \succ_{3} r$ and $L(r) \succ_{2} L^{\prime}(q)$ then at node $\square 1$, he has the choice between $L(r)$ obtained when moving up (subtree $T_{1}$ ) and $L^{\prime}(q)$ obtained when moving down (subtree $T_{2}$ ). Since $L(r) \succ_{1} L^{\prime}(q)$ then he will choose to move up, that is to choose to choose in subtree $T_{1}$, hence he is Information Averse.

A similar reasoning with type 2-preference relation leads to the same conclusion. This completes the proof of Lemma 1.

We complete the proof of Theorem 1 a) by taking $\underset{2}{\succsim}$ and $\underset{3}{\succsim}$ as subrelations of $\succsim$ a preference relation over $\left\{L(r), L^{\prime}(q), r, q\right\}$. 


\section{B Proof of Theorem $1 \mathrm{~b}$ )}

Lemma 2 Let a DM have choice functions $C_{i}, i=1,2,3$, when choosing over the sets $X_{1}=\left\{T_{1}, T_{2}, L(r), L^{\prime}(q)\right\}, X_{2}=\left\{L(r), L^{\prime}(q)\right\}$ and $X_{3}=\{r, q\}$. For strategically equivalent subtrees $\mathbf{T}_{1}$ and $\mathbf{T}_{2}$, the following two conditions are equivalent.

1. The DM is Information Loving (or Seeking).

2. The DM s preferences at nodes $\square 1, \square 2$ and $\square 3$ denoted respectively $\underset{1}{\succsim}$, $\underset{2}{\succsim}$ and $\underset{3}{\succsim}$ are either of

- Type 3, that is

$3.1 L(r) \succ L^{\prime}(q)$

3.2 $L^{\prime}(q) \succ_{2} L(r)$

$3.3 r \succ_{3} q$

or of

- Type 4, that is

$4.1 L^{\prime}(q) \succ L(r)$

4.2 $L(r) \succ_{2} L^{\prime}(q)$

$4.3 q \succ_{3} r$

Proof.

- $(1) \Longrightarrow(2)$

If the DM is Information Loving, then he chooses to choose between the lotteries $r$ and $q$ directly rather than in a game (a compound lottery), that is

$$
C_{1}\left\{C_{2}\left\{L(r), L^{\prime}(q)\right\} ;\left[\lambda, C_{3}\{r, q\} ; 1-\lambda, q^{\prime}\right]\right\}=\left[\lambda, C_{3}\{r, q\} ; 1-\lambda, q^{\prime}\right]
$$

Let $\underset{1}{\succsim}$ be the DMs (revealed) preference relation de ned over the set $X_{1}$. The DM strictly prefers $(\succ)$ at node $\square 1,\left[\lambda, C_{3}\{r, q\} ; 1-\lambda, q^{\prime}\right]$ to $C_{2}\left\{L(r), L^{\prime}(q)\right\}$, that is:

- $\underline{\text { At node } \square 1},\left[\lambda, C_{3}\{r, q\} ; 1-\lambda, q^{\prime}\right] \succ_{1} C_{2}\left\{L(r), L^{\prime}(q)\right\}$.

- At node $\square 2, C_{2}\left\{L(r), L^{\prime}(q)\right\}$ gives three possibilities of choice, so is $C_{3}\{r, q\}$ at node $\square$ 3. That leads to 9 cases. Let us represent these cases by the following graph: 


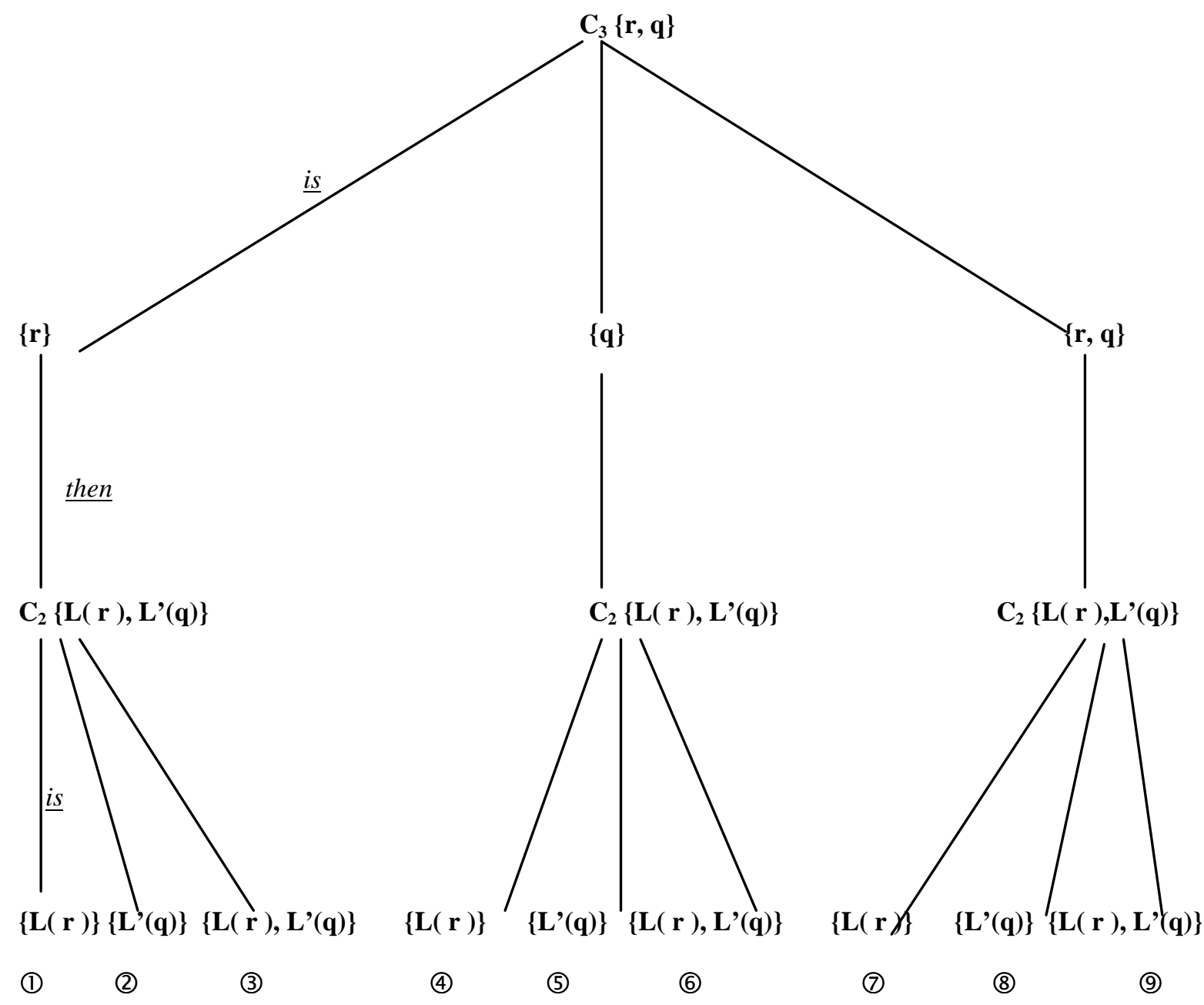

Among these cases, only two (cases 2 and 4) are logically possible. Let us consider these two cases.

* Case 2:

Since $C_{3}\{r, q\}=\{r\}$, then at node $\square 3, r_{3} q$. Since $C_{2}\left\{L(r), L^{\prime}(q)\right\}=\left\{L^{\prime}(q)\right\}$, then at node $\square 2, L^{\prime}(q) \succ_{2} L(r)$. Therefore, $\left[\lambda, C_{3}\{r, q\} ; 1-\lambda, q^{\prime}\right] \succ_{1} C_{2}\left\{L(r), L^{\prime}(q)\right\}$ is equivalent to $\left[\lambda, r ; 1-\lambda, q^{\prime}\right] \succ_{1} L^{\prime}(q)$, that is $L(r) \succ L^{\prime}(q)$.

That is type 3 .

* Case $4:$

Since $C_{3}\{r, q\}=\{q\}$, then at node $\square 3, q \succ r$. Since $C_{2}\left\{L(r), L^{\prime}(q)\right\}=\{L(r)\}$, then at node $\square 2, L(r) \succ_{2} L^{\prime}(q)$. Therefore, $\left[\lambda, C_{3}\{r, q\} ; 1-\lambda, q^{\prime}\right] \succ_{1} C_{2}\left\{L(r), L^{\prime}(q)\right\}$ is equivalent to $\left[\lambda, q ; 1-\lambda, q^{\prime}\right] \succ_{1} L(r)$, that is $L^{\prime}(q) \succ_{1} L(r)$.

That is type 4 . 
The other cases $1,3,5,6,7,8$ and 9 are logically impossible.

- $(2) \Longrightarrow(1)$

Let a DM exhibiting a pro le of preference relations of type 3 (the reasoning is the same for type 4), then since $r \succ_{3} q$ and $L^{\prime}(q) \succ_{2} L(r)$, then at node $\square 1$ he has the choice between the compound lottery $L^{\prime}(q)$ obtained by moving up (subtree $T_{1}$ ) and the compound lottery $L(r)$ obtained by moving down (subtree $T_{2}$ ). Since $L(r) \succ L^{\prime}(q)$ then the DM will choose at node $\square 1$ to move down, that is to choose to choose in subtree $T_{2}$. Hence, he is Information Seeking. This completes the proof of Lemma 2.

We complete the proof of Theorem 1 b) by taking $\underset{2}{\succsim}$ and $\underset{3}{\succsim}$ as subrelations of $\succsim$ a preference relation over $\left\{L(r), L^{\prime}(q), r, q\right\}$. 


\section{Proof of Theorem 1 c)}

Lemma 3 Let a DM have choice functions $C_{i}, i=1,2,3$, when choosing over the sets $X_{1}=\left\{T_{1}, T_{2}, L(r), L^{\prime}(q)\right\}, X_{2}=\left\{L(r), L^{\prime}(q)\right\}$ and $X_{3}=\{r, q\}$. For strategically equivalent subtrees $\mathbf{T}_{1}$ and $\mathbf{T}_{2}$, the following two conditions are equivalent.

1. The DM is Information Neutral.

2. The DM s preferences at nodes $\square 1, \square 2$ and $\square 3$ denoted respectively $\underset{1}{\underset{\sim}{~}} \underset{2}{\succsim}$ and $\underset{3}{\succsim}$ are one of the following 9 types:

- Type A:

A.1 $L(r) \underset{1}{\sim} L^{\prime}(q)$

A.2 $L(r) \succ_{2} L^{\prime}(q)$

A.3 $q \succ_{3} r$

- Type B:

$B .1 L^{\prime}(q) \underset{1}{\sim} L^{\prime}(q)$ and $L(r) \underset{1}{\sim} L^{\prime}(q)$

B.2 $L(r) \underset{2}{\sim} L^{\prime}(q)$

$B .3 q \succ_{3} r$

- Type $C$ :

C.1 $L^{\prime}(q) \underset{1}{\sim} L^{\prime}(q)$

C.2 $L^{\prime}(q) \succ_{2} L(r)$

C.3 $q \succ_{3} r$

- Type D:

$D .1 L(r) \underset{1}{\sim} L(r)$

D.2 $L(r) \succ_{2} L^{\prime}(q)$

$D .3 r \succ_{3} q$

- Type E:

E.1 $L(r) \underset{1}{\sim} L(r)$ and $L(r) \underset{1}{\sim} L^{\prime}(q)$

E.2 $L(r) \underset{2}{\sim} L^{\prime}(q)$

E.3 $r_{3} q$

- Type F:

F.1 $L(r) \underset{1}{\sim} L^{\prime}(q)$

F.2 $L^{\prime}(q) \succ_{2} L(r)$

F.3 $r_{3} q$ 
- Type $G$ :

G.1 $L(r) \underset{1}{\sim} L(r)$ and $L(r) \underset{1}{\sim} L^{\prime}(q)$

G.2 $L(r) \succ_{2} L^{\prime}(q)$

G.3 $\underset{3}{\sim} q$

- Type $H$ :

H.1 $L(r) \underset{1}{\sim} L(r)$ and $L^{\prime}(q) \underset{1}{\sim} L^{\prime}(q)$ and $L(r) \underset{1}{\sim} L^{\prime}(q)$

$H .2 L(r) \underset{2}{\sim} L^{\prime}(q)$

H.3 $r \underset{3}{\sim} q$

- $\underline{\text { Type I: }}$

I.1 $L^{\prime}(q) \underset{1}{\sim} L^{\prime}(q)$ and $L(r) \underset{1}{\sim} L^{\prime}(q)$

I.2 $L^{\prime}(q) \succ_{2} L(r)$

$1.3 r \underset{3}{\sim} q$

\section{Proof.}

- $(1) \Longrightarrow(2)$

If the DM is Information Neutral, then he is indifferent at node $\square 1$ between going up (subtree $T_{1}$ ) and going down (subtree $T_{2}$ ). That is he is indifferent between choosing between the compound lotteries $L(r)$ and $L^{\prime}(q)$ or choosing between the lotteries $r$ and $q$, that is he is indifferent between getting $r$ or $q$ directly or through a game (a compound lottery). Formally,

$C_{1}\left\{C_{2}\left\{L(r), L^{\prime}(q)\right\} ;\left[\lambda, C_{3}\{r, q\} ; 1-\lambda, q^{\prime}\right]\right\}=\left\{C_{2}\left\{L(r), L^{\prime}(q)\right\} ;\left[\lambda, C_{3}\{r, q\} ; 1-\lambda, q^{\prime}\right]\right\}$

Let $\underset{1}{\succsim}$ be the DMs (revealed) preference relation de ned over $X_{1}$. The DM is indifferent $\left(\begin{array}{c}\sim \\ 1\end{array}\right)$ at node $\square 1$ between $C_{2}\left\{L(r), L^{\prime}(q)\right\}$ and $\left[\lambda, C_{3}\{r, q\} ; 1-\lambda, q^{\prime}\right]$, that is:

- At node $\square 1, C_{2}\left\{L(r), L^{\prime}(q)\right\} \underset{1}{\sim}\left[\lambda, C_{3}\{r, q\} ; 1-\lambda, q^{\prime}\right]$.

- At node $\square 2: C_{2}\left\{L(r), L^{\prime}(q)\right\}$ gives also three possibilities of choice, so is $C_{3}\{r, q\}$ at node $\square$ 3. That leads to 9 cases. Let us represent these cases by the following graph: 


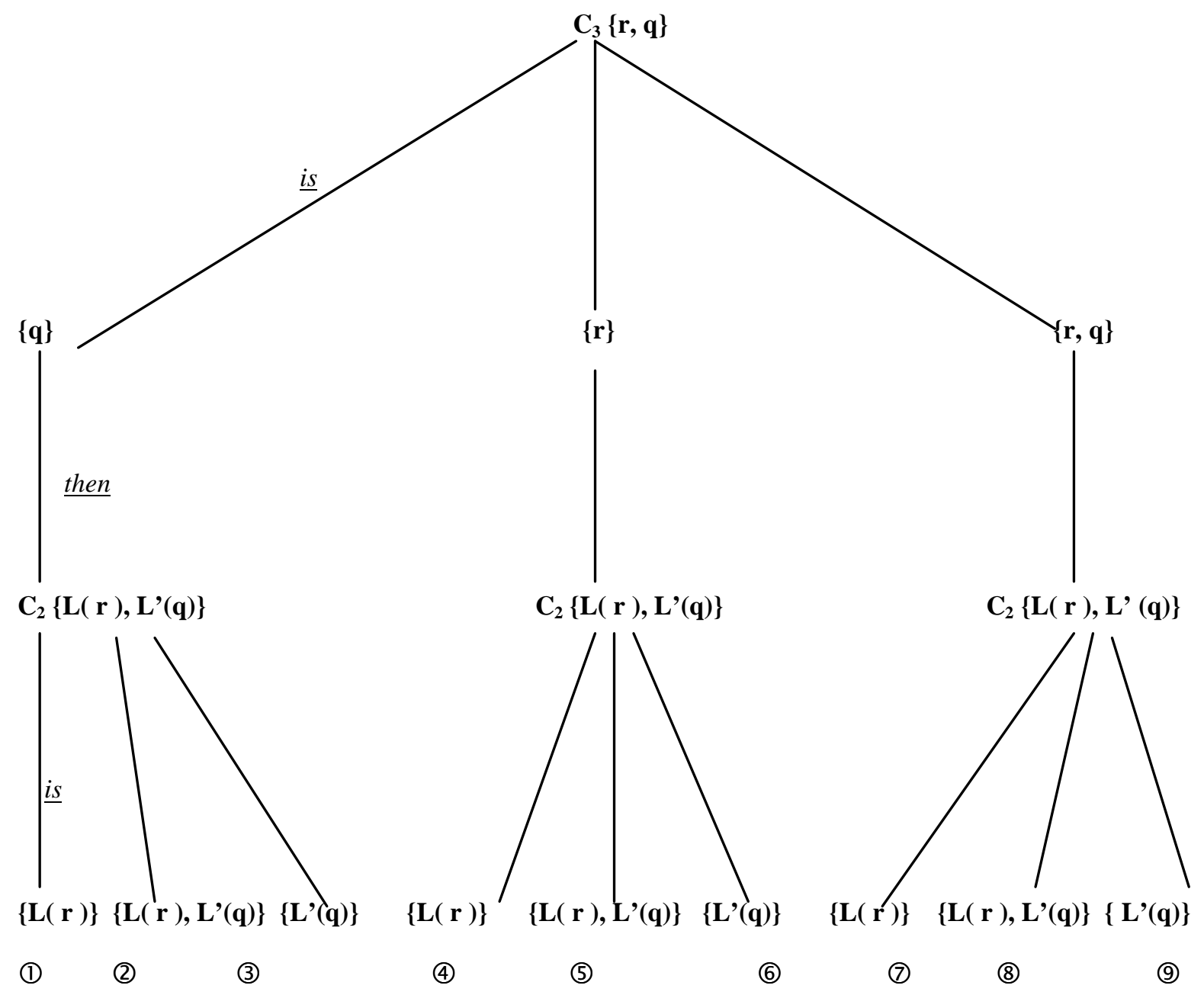

* In case $1:$

$C_{3}\{r, q\}=\{q\}$, that is at node $\square 3, q \succ_{3} r . C_{2}\left\{L(r), L^{\prime}(q)\right\}=\{L(r)\}$, that is at node $\square 2, L(r) \succ_{2} L^{\prime}(q)$. Therefore, $C_{2}\left\{L(r), L^{\prime}(q)\right\} \underset{1}{\sim}\left[\lambda, C_{3}\{r, q\} ; 1-\lambda, q^{\prime}\right]$ is equivalent to $L(r) \underset{1}{\sim}\left[\lambda, q ; 1-\lambda, q^{\prime}\right]$, that is $L(r) \sim L^{\prime}(q)$.

That is type A.

* In case 2:

$C_{3}\{r, q\}=\{q\}$, that is at node $\square 3, q \succ_{3} r . C_{2}\left\{L(r), L^{\prime}(q)\right\}=\left\{L(r), L^{\prime}(q)\right\}$, that is at node $\square 2, L(r) \underset{2}{\sim} L^{\prime}(q)$. Therefore, $C_{2}\left\{L(r), L^{\prime}(q)\right\} \underset{1}{\sim}\left[\lambda, C_{3}\{r, q\} ; 1-\lambda, q^{\prime}\right]$ is equivalent to $L(r) \underset{1}{\sim}\left[\lambda, q ; 1-\lambda, q^{\prime}\right]$ and $L^{\prime}(q) \underset{1}{\sim}\left[\lambda, q ; 1-\lambda, q^{\prime}\right]$, that is $L(r) \underset{1}{\sim} L^{\prime}(q)$ and $L^{\prime}(q) \underset{1}{\sim} L^{\prime}(q)$.

That is type B.

* In case 3: 
$C_{3}\{r, q\}=\{q\}$, that is at node $\square 3, q \succ r . C_{2}\left\{L(r), L^{\prime}(q)\right\}=\left\{L^{\prime}(q)\right\}$, that is at node

$2, L^{\prime}(q) \succ_{2} L(r)$. Therefore, $C_{2}\left\{L(r), L^{\prime}(q)\right\} \underset{1}{\sim}\left[\lambda, C_{3}\{r, q\} ; 1-\lambda, q^{\prime}\right]$ is equivalent to $L^{\prime}(q) \underset{1}{\sim}\left[\lambda, q ; 1-\lambda, q^{\prime}\right]$, that is $L^{\prime}(q) \underset{1}{\sim} L^{\prime}(q)$.

That is type $\mathrm{C}$.

* In case $4:$

$C_{3}\{r, q\}=\{r\}$, that is at node $\square 3, r \succ q . C_{2}\left\{L(r), L^{\prime}(q)\right\}=\{L(r)\}$, that is at node

2, $L(r) \succ_{2} L^{\prime}(q)$. Therefore, $C_{2}\left\{L(r), L^{\prime}(q)\right\} \underset{1}{\sim}\left[\lambda, C_{3}\{r, q\} ; 1-\lambda, q^{\prime}\right]$ is equivalent to

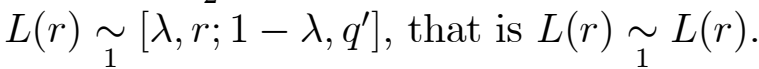

That is type $\mathrm{D}$.

* In case $5:$

$C_{3}\{r, q\}=\{r\}$, that is at node $\square 3, r \succ_{3} q . C_{2}\left\{L(r), L^{\prime}(q)\right\}=\left\{L(r), L^{\prime}(q)\right\}$, that is at node $\square 2, L(r) \underset{2}{\sim} L^{\prime}(q)$. Therefore, $C_{2}\left\{L(r), L^{\prime}(q)\right\} \underset{1}{\sim}\left[\lambda, C_{3}\{r, q\} ; 1-\lambda, q^{\prime}\right]$ is equivalent to $L(r) \underset{1}{\sim}\left[\lambda, r ; 1-\lambda, q^{\prime}\right]$ and $L^{\prime}(q) \underset{1}{\sim}\left[\lambda, r ; 1-\lambda, q^{\prime}\right]$, that is $L(r) \underset{1}{\sim} L(r)$ and $L^{\prime}(q) \underset{1}{\sim} L(r)$.

That is type E.

* In case $6:$

$C_{3}\{r, q\}=\{r\}$, that is at node $\square 3, r_{3} q . C_{2}\left\{L(r), L^{\prime}(q)\right\}=\left\{L^{\prime}(q)\right\}$, that is at node

$2, L^{\prime}(q) \succ_{2} L(r)$. Therefore, $C_{2}\left\{L(r), L^{\prime}(q)\right\} \underset{1}{\sim}\left[\lambda, C_{3}\{r, q\} ; 1-\lambda, q^{\prime}\right]$ is equivalent to $L^{\prime}(q) \sim\left[\lambda, r ; 1-\lambda, q^{\prime}\right]$, that is $L^{\prime}(q) \underset{1}{\sim} L(r)$.

That is type $\mathrm{F}$.

* In case 7:

$C_{3}\{r, q\}=\{r, q\}$, that is at node $\square 3, r \underset{3}{\sim} q . C_{2}\left\{L(r), L^{\prime}(q)\right\}=\{L(r)\}$, that is at node $\square 2, L(r) \succ_{2} L^{\prime}(q)$. Therefore, $C_{2}\left\{L(r), L^{\prime}(q)\right\} \sim\left[{ }_{1}\left[\lambda, C_{3}\{r, q\} ; 1-\lambda, q^{\prime}\right]\right.$ is equivalent to $L(r) \underset{1}{\sim}\left[\lambda, r ; 1-\lambda, q^{\prime}\right]$ and $L(r) \underset{1}{\sim}\left[\lambda, q ; 1-\lambda, q^{\prime}\right]$, that is $L(r) \underset{1}{\sim} L(r)$ and $L(r) \underset{1}{\sim} L^{\prime}(q)$.

That is type $\mathrm{G}$.

* In case 8:

$C_{3}\{r, q\}=\{r, q\}$, that is at node $\square 3, r \underset{3}{\sim} q$.

$C_{2}\left\{L(r), L^{\prime}(q)\right\}=\left\{L(r), L^{\prime}(q)\right\}$, that is at node $\square 2, L(r) \underset{2}{\sim} L^{\prime}(q)$. Therefore, $C_{2}\left\{L(r), L^{\prime}(q)\right\} \sim\left[\lambda, C_{3}\{r, q\} ; 1-\lambda, q^{\prime}\right]$ is equivalent to $L(r) \sim\left[\lambda, r ; 1-\lambda, q^{\prime}\right], L(r) \sim$ $\left[\lambda, q ; 1-\lambda, q^{\prime}\right], L^{\prime}(q) \sim\left[\lambda, r ; 1-\lambda, q^{\prime}\right]$ and $L^{\prime}(q) \sim\left[\lambda, q ; 1-\lambda, q^{\prime}\right]$, that is $L(r) \sim L(r)$, $L(r) \underset{1}{\sim} L^{\prime}(q)$ and $L^{\prime}(q) \underset{1}{\sim} L^{\prime}(q)$.

That is type $\mathrm{H}$.

* In case 9:

$C_{3}\{r, q\}=\{r, q\}$, that is at node $\square 3, r \underset{3}{\sim} q$.

$C_{2}\left\{L(r), L^{\prime}(q)\right\}=\left\{L^{\prime}(q)\right\}$, that is at node $\square 2, L^{\prime}(q) \succ_{2} L(r)$. 
Therefore, $C_{2}\left\{L(r), L^{\prime}(q)\right\} \underset{1}{\sim}\left[\lambda, C_{3}\{r, q\} ; 1-\lambda, q^{\prime}\right]$ is equivalent to $L^{\prime}(q) \underset{1}{\sim}\left[\lambda, r ; 1-\lambda, q^{\prime}\right]$ and $L^{\prime}(q) \underset{1}{\sim}\left[\lambda, q ; 1-\lambda, q^{\prime}\right]$, that is $L^{\prime}(q) \underset{1}{\sim} L(r)$ and $L^{\prime}(q) \underset{1}{\sim} L^{\prime}(q)$.

That is type I.

- $(2) \Longrightarrow(1)$

Do the same reasoning as in the proofs of Lemmas 1 and 2. This completes the proof of Lemma 3.

We complete the proof of Theorem 1 c) by taking $\underset{2}{\succsim}$ and $\underset{3}{\succsim}$ as subrelations of $\succsim$ a preference relation over $\left\{L(r), L^{\prime}(q), r, q\right\}$. 


\section{Proof of lemma 4 and 5}

Lemma 4 If DM is information averse (respectively information lover or information neutral) for the decision problem $\left.\mathbb{P}=\left\{\lambda r+(1-\lambda) q^{\prime}, \lambda q+(1-\lambda) q^{\prime}\right\}, \forall \lambda \in\right] 0,1[, \forall$ lotteries $r$, $q$ and $q^{\prime}$ then he is information averse for decision problem $\mathbb{P}^{\prime}=\left\{\lambda_{1} r+\sum_{j=2}^{m} \lambda_{j} q_{j}^{\prime}, \lambda_{1} q+\sum_{j=2}^{m} \lambda_{j} q_{j}^{\prime}\right\}$.

Proof.

It is obvious. Indeed in both cases $\mathbb{P}$ and $\mathbb{P}^{\prime}$, attitude towards information is determined at decision nodes $\square 1, \square 2$ and $\square 3$, (See gure 3 in the body text and the gure below).

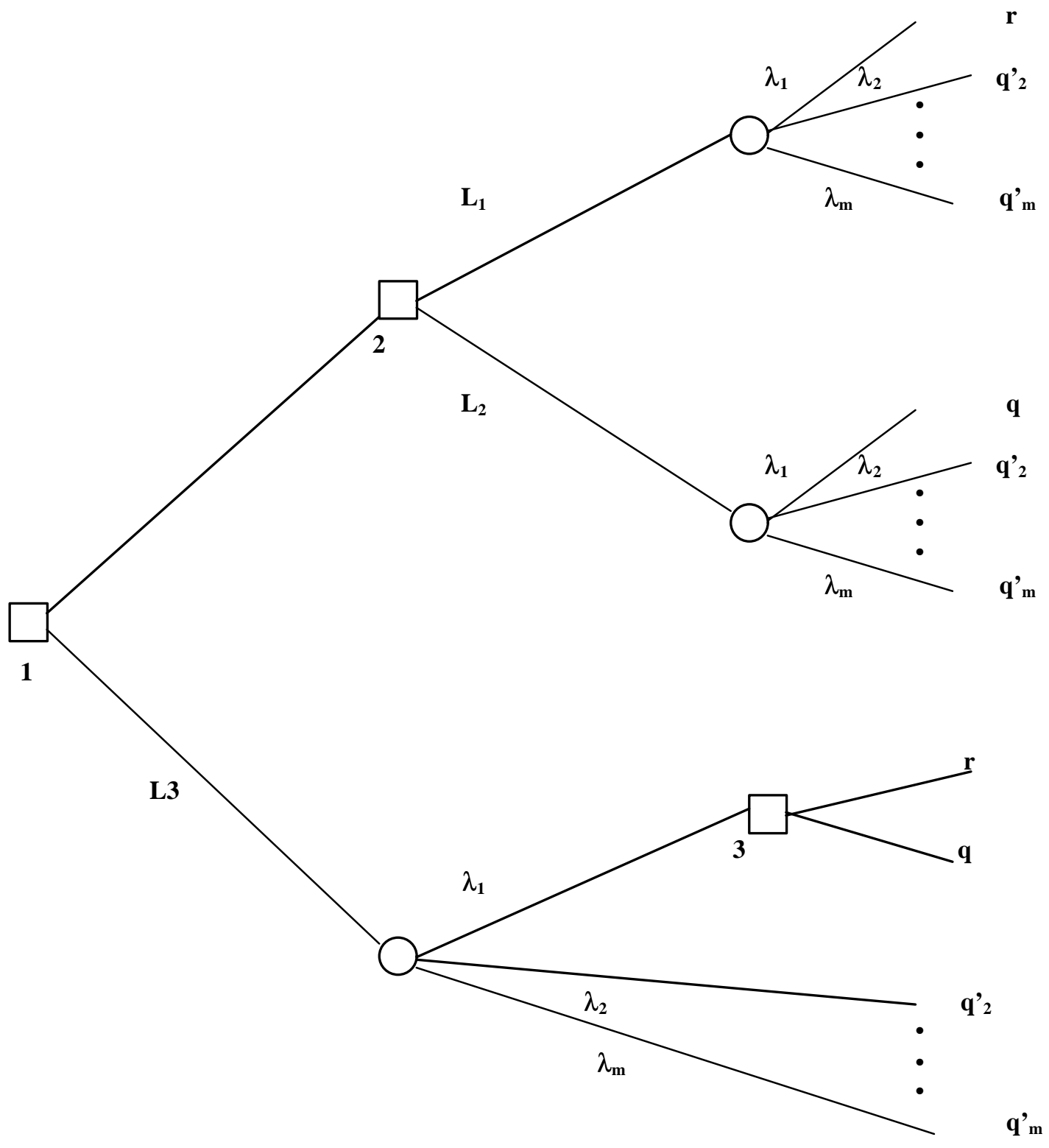


Lemma 5 The following two conditions are equivalent.

1. $\forall \lambda \in] 0,1\left[, \forall\right.$ lotteries $r, q$ and $q^{\prime}, D M$ is information averse (respectively information lover or information neutral) for the decision problem

$$
\mathbb{P}=\left\{\lambda r+(1-\lambda) q^{\prime}, \lambda q+(1-\lambda) q^{\prime}\right\}
$$

2. The DM is information averse (respectively information lover, information neutral).

\section{Proof.}

$(2) \Longrightarrow(1)$ is immediate.

$(1) \Longrightarrow(2)$ The strategy to prove this implication will be the following. We want to prove that $(1) \Longrightarrow(2)$, that is $: \forall \mathfrak{C}_{i}, \forall T, T^{\prime} \in \mathfrak{C}_{i}$ :

$\cdot I(T)>I\left(T^{\prime}\right) \Longrightarrow T P_{R_{i}} T^{\prime}$

$\cdot I(T)=I\left(T^{\prime}\right) \Longrightarrow T I_{R_{i}} T^{\prime}$

Assuming H2, we have only to prove that $(1) \Longrightarrow\left[\forall \mathfrak{C}_{i}, \forall T, T^{\prime} \in \mathfrak{C}_{i}, I(T)>I\left(T^{\prime}\right) \Longrightarrow\right.$ $\left.T P_{R_{i}} T^{\prime}\right]$. Let us call this implication $(*)$. Let $\mathfrak{C}_{i}$ be a class of strategically equvalent decision trees. $\mathfrak{C}_{i}$ includes a nite number of trees. Let us de ne the following binary relation is more informative than denoted $\mathfrak{I}$ and de ned by : $T \mathfrak{I} T^{\prime}$ if $I(T) \geq I\left(T^{\prime}\right)$. It is obvious that $\mathfrak{I}$ is a preorder (complete and transitive). Let $\mathfrak{C}_{i} / \mathfrak{I}$ be the quotient set and let $\mathfrak{I}^{*}$ be the quotient order. Let us numbered the equivalent classes in the sense of $\mathfrak{I}$ such that $C_{i}^{*}$ is the class having elements $T$ with $I(T)=i-1$ where $\mathrm{i}=1$ to n. To show (*) it is sufficient to show that : (1) $\Longrightarrow\left[\forall \mathfrak{C}_{i}, \forall T \in C_{t}^{*}, \forall T^{\prime} \in C_{t^{\prime}}^{*}, t>t^{\prime} \Longrightarrow T P_{R_{i}} T^{\prime}\right]$. $C_{1}^{*}$ and $C_{2}^{*}$ include only one decision trees. We will divide our proof into two parts. In the rst part (PART I) of the proof, we will show that $(1) \Longrightarrow\left[\forall \mathfrak{C}_{i}, T \in C_{1}^{*}, T^{\prime} \in C_{2}^{*}, t>\right.$ $\left.t^{\prime} \Longrightarrow T P_{R_{i}} T^{\prime}\right]$. Let us call this implication $(* *)$. In the second part (PART II) of the proof, we will show that one can restrict to $C_{1}^{*}$ and $C_{2}^{*}$ without loss of generality : $(* *) \Longrightarrow\left[\forall \mathfrak{C}_{i}, \forall T \in C_{t}^{*}, \forall T^{\prime} \in C_{t^{\prime}}^{*}, t>t^{\prime} \Longrightarrow T P_{R_{i}} T^{\prime}\right]$

\section{PART I}

Let $\mathfrak{C}_{i}$ be an equivalence class of $\Pi / S E$ and $\mathbb{P}_{i}$ be its associated decision problem. The opportunity set $\mathbb{O}_{i}$ is a set of lotteries. Let us recall that these lotteries have as consequences the set of endpoints of the decision trees belonging to $\mathfrak{C}_{i}$. These lotteries have a special form since the decision trees are strategically equivalent. They have the same probability distribution over their consequences (which are however different from a lottery to another). Thus if we take two lotteries $L$ and $L^{\prime}$ in $\mathbb{O}_{i}$, they will reach their consequences at the same number of stages, say n. We will say that $L$ and $L^{\prime}$ are $n$-stages lotteries. Moreover, lotteries $L$ and $L^{\prime}$ have (at least) a common consequence (otherwise the decision problem cannot be modeled by strategically equivalent decision trees). Since the decision trees are supposed to be nite, so are the opportunity sets.

- If $\operatorname{Card} \mathbb{O}_{i}=2$ that is if $\mathbb{O}_{i}$ includes only two lotteries $L$ and $L^{\prime}$ then they are necessarily of the form : $L=\lambda_{1} r+\sum_{j=2}^{m} \lambda_{j} q_{j}^{\prime}, L^{\prime}=\lambda_{1} q+\sum_{j=2}^{m} \lambda_{j} q_{j}^{\prime}$ where $r, q$ and the $q_{j}^{\prime}$ are lotteries, $\mathrm{r}$ and $\mathrm{q}$ are different, $\mathrm{r}$ and $\mathrm{q}$ are $\mathrm{n}-1$ stages lotteries if $L$ and $L^{\prime}$ are n-stages lotteries. In such a case, we apply the above lemma 4 and get the required result. 
- If $\operatorname{Card} \mathbb{O}_{i}=3$ that is if $\mathbb{O}_{i}$ includes three lotteries then they are necessarily of the form $L 1=\lambda_{1} r+\sum_{j=2}^{m} \lambda_{j} q_{j}^{\prime}, L 2=\lambda_{1} q+\sum_{j=2}^{m} \lambda_{j} q_{j}^{\prime}$ and $L 3=\lambda_{1} z+\sum_{j=2}^{m} \lambda_{j} q_{j}^{\prime}$, where $\mathrm{r}, \mathrm{q}$ and $\mathrm{z}$ are pairwise different, $\mathrm{r}, \mathrm{q}$ and $\mathrm{z}$ are $\mathrm{n}-1$ stages lotteries if $L 1, L 2$ and $L 3$ are $\mathrm{n}$ stages lotteries. Let us consider the following three decisions problems $\mathbb{P} 1=\{L 1, L 2\}, \mathbb{P} 2=\{L 2, L 3\}, \mathbb{P} 3=\{L 1, L 3\}$. Since the agent is information averse for the decision problem $\mathbb{P}=\left\{\lambda r+(1-\lambda) q^{\prime}, \lambda q+(1-\lambda) q^{\prime}\right\}$, $\forall \lambda \in] 0,1\left[, \forall\right.$ lotteries $r, q$ and $q^{\prime}$ then he is, according to Lemma 4, information averse over $\mathbb{P} 1, \mathbb{P} 2$, and $\mathbb{P} 3$ (see the case with $\operatorname{Card} \mathbb{O}_{i}=2$ ). Let us display the revealed preference of the $\mathrm{DM}$ when confronted with $\mathbb{P} 1, \mathbb{P} 2$, and $\mathbb{P} 3$, using Lemma 1.

Over $\mathbb{P} 1$, the possible preferences are :

Con guration 1.1. $L 1 \succ_{1} L 2, L 1 \succ_{2} L 2, q \succ_{3} r$ or,

Con guration 1.2. $L 2 \succ_{1} L 1, L 2 \succ_{2} L 1, r \succ_{3}^{\succ} q$.

Over $\mathbb{P} 2$, the possible preferences are :

Con guration 2.1. $L 3 \succ_{1} L 2, L 3 \succ_{2} L 2, q \succ_{3} z$ or,

Con guration 2.2. $L 2 \succ_{1}^{1} L 3, L 2 \underset{2}{\succ} L 3, z \underset{3}{\succ} q$.

Over $\mathbb{P} 3$, the possible preferences are :

Con guration 3.1. $L 1 \succ L 3, L 1 \succ_{2} L 3, r \succ_{3} z$ or,

Con guration 3.2. $L 3 \succ_{1}^{1} L 1, L 3 \succ_{2}^{2} L 1, z \succ_{3}^{3} r$.

Let us show that the preferences displayed over $\mathbb{P} 1, \mathbb{P} 2$, and $\mathbb{P} 3$ when the DM is information averse over these sets, lead to the conclusion that DM is information averse over $\{L 1, L 2, L 3\}$. There are $2 \times 2 \times 2=8$ cases. But 4 are logically impossible because they violate the decisiveness assumption of the choice functions. We will not enumerate all the 8 cases, let us give just two cases (one possible and one impossible).

Con guration 1.1 + Con guration 2.1 + Con guration 3.1:L1 $\succ_{1} L 2, L 1 \succ_{2}$ $L 2, q \succ_{3} r$ and $L 3 \succ_{1} L 2, L 3 \succ_{2} L 2, q \succ_{3} z$ and $L 1 \succ_{1} L 3, L 1 \succ_{2} L 3, r \succ_{3} z$. In the decision problem $\{L 1, L 2, L 3\}$, the DM has the choice at decision node $\square 1$ between the following trees : 


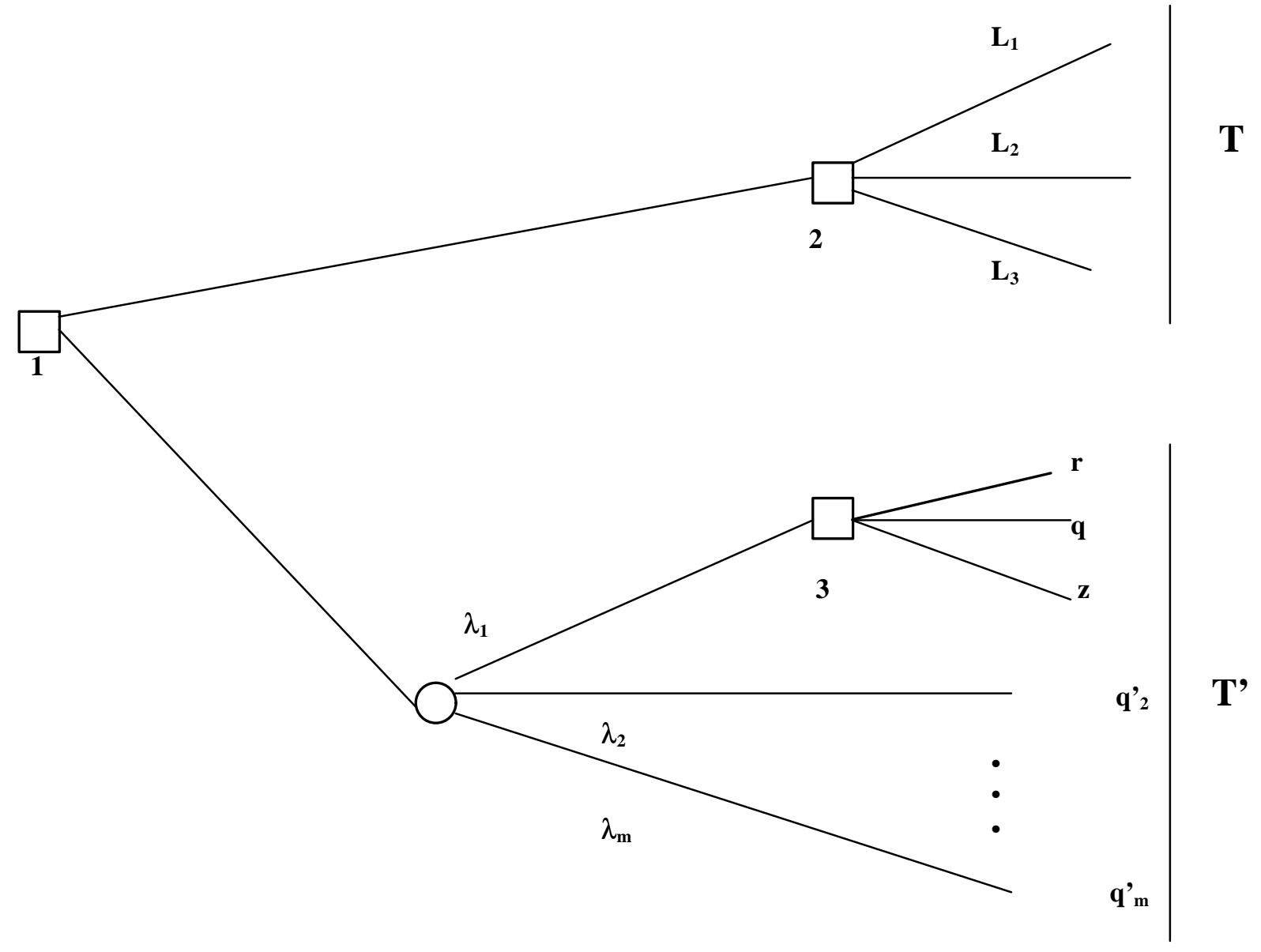

Since $q \succ_{3} r, q \succ_{3} z$ and $r \succ_{3} z$, then at $\square 3$ the DM will choose lottery q. Then over decision tree T , lottery L2 will be choosen. Over decision tree T, since we have $L 1 \succ_{2} L 2$, $L 3 \succ_{2} L 2, L 1 \succ_{2} L 3$, then lottery L1 will be choosen at decision node $\square 2$. Well at $\square 1$, we have $L 1 \succ L 2$ then DM will prefer to choose over $\mathrm{T}$ in order to get $\mathrm{L} 1$. Thus he is information averse for the decision problem $\{L 1, L 2, L 3\}$.

Con guration 1.1 + Con guration 2.2 + Con guration 3.1 is impossible because it violates the decisiveness hypothesis of the choice functions. Indeed $q \succ_{3} r, r \succ_{3} z$ and $z \succ_{3} q$, leads to a cycle in the preference $\succ_{3}$ and to the conclusion that $C_{3}\{q, r, z\}=\varnothing$.

If $k \geq 4$, then the lotteries can be of different shapes. For instance when $k=4$ then two possible shapes are the following :

Either (shape 1) : $L 1=\lambda_{1} r+\sum_{j=2}^{m} \lambda_{j} q_{j}^{\prime}, L 2=\lambda_{1} q+\sum_{j=2}^{m} \lambda_{j} q_{j}^{\prime}, L 3=\lambda_{1} z+$ $\sum_{j=2}^{m} \lambda_{j} q_{j}^{\prime}$, and $L 4=\lambda_{1} a+\sum_{j=2}^{m} \lambda_{j} q_{j}^{\prime}$, where r, q, a and $\mathrm{z}$ are pairwise different, r, $\mathrm{q}$, a and $\mathrm{z}$ are $\mathrm{n}-1$ stages lotteries if $L 1, L 2, L 3$ and $L 4$ are $\mathrm{n}$ stages lotteries. 


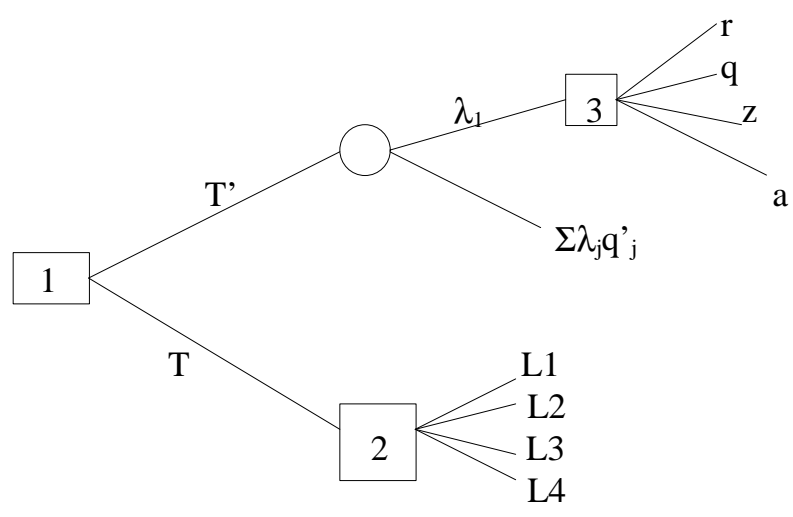

Or (shape 2) : $L 1=\lambda_{1} r+\lambda_{2} z+\sum_{j=3}^{m} \lambda_{j} q_{j}^{\prime}, L 2=\lambda_{1} r+\lambda_{1} a+\sum_{j=3}^{m} \lambda_{j} q_{j}^{\prime}, L 3=$ $\lambda_{1} q+\lambda_{1} z+\sum_{j=3}^{m} \lambda_{j} q_{j}^{\prime}$, and $L 4=\lambda_{1} q+\lambda_{1} a+\sum_{j=3}^{m} \lambda_{j} q_{j}^{\prime}$, where r, q, a and $\mathrm{z}$ are pairwise different, r, q, a and z are n- 1 stages lotteries if $L 1, L 2, L 3$ and $L 4$ are $\mathrm{n}$ stages lotteries.

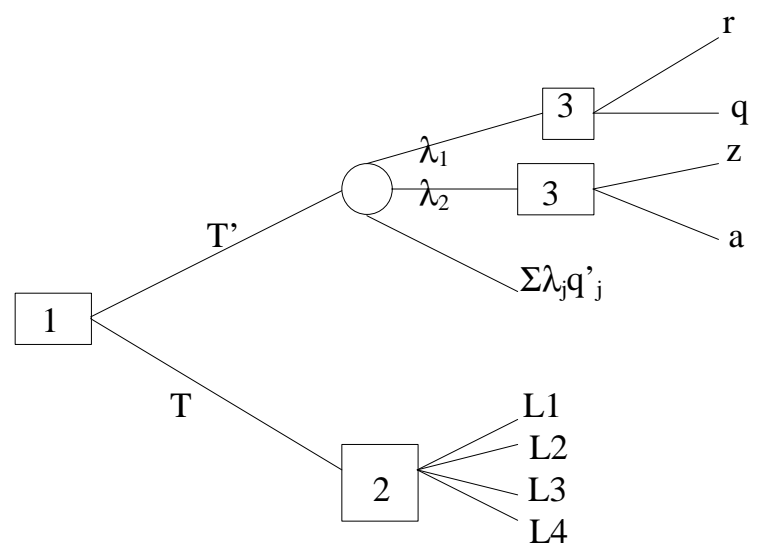

The two shapes lead to two different representations (see the above gures).

Generally speaking when $\operatorname{Card} \mathbb{O}_{i}=k \geq 2$, the number of possible shapes is $\prod_{i=1}^{l} e_{i}$ where $k=\prod_{i=1}^{l}\left(k_{i}\right)^{e_{i}}$, the $k_{i}$ are prime numbers and the $e_{i}$ are strictly positive integers. Let 
us take a shape and its associated representation (see the below gure). We will number without loss of generality the different decision nodes as indicated in the below gure.

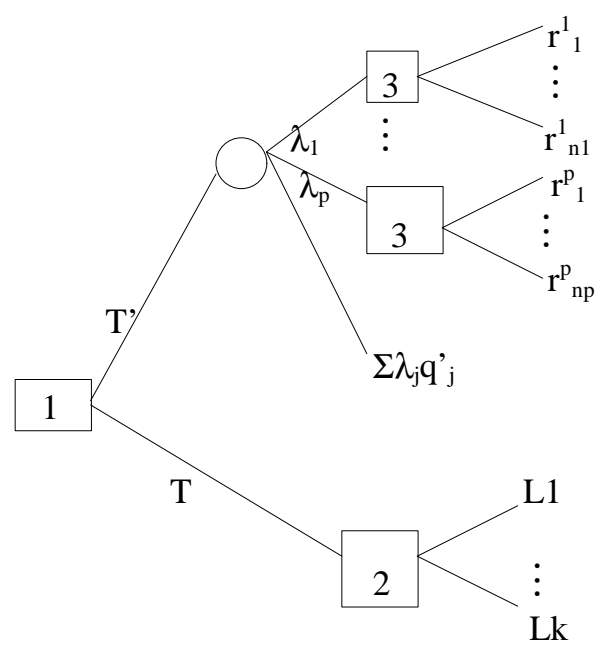

Figure PG

It is easy to see that the $\mathrm{k}$ lotteries of $\mathbb{O}_{i}$ have the following shape : $L_{s}=\lambda_{1} r_{i_{1}}^{1}+$ $\lambda_{2} r_{i_{2}}^{2}+\ldots . .+\lambda_{p} r_{i_{p}}^{p}+\sum_{j=p+1}^{m} \lambda_{j} q_{j}^{\prime}$ with $i_{1}=1$ to $n_{1} ; i_{2}=1$ to $n_{2} ; \ldots \ldots . ., i_{p}=1$ to $n_{p}$; and $s=i_{1} \times i_{2} \times \ldots \times i_{p}, s=1$ to $k$.

There are $C_{k}^{2}$ possible paire of lotteries. But all these paires are not a decision problem in the sense of de nition 1, that is they are not associated with an equivalent class including some strategically equivalent decision trees. For instance : if we take $L=\lambda_{1} r_{1}^{1}+$ $\lambda_{2} r_{1}^{2}+\lambda_{3} r_{1}^{3}+\ldots .+\lambda_{p} r_{1}^{p}+\sum_{j=p+1}^{m} \lambda_{j} q_{j}^{\prime}$ and $L^{\prime}=\lambda_{1} r_{2}^{1}+\lambda_{2} r_{2}^{2}+\lambda_{3} r_{1}^{3}+\ldots .+\lambda_{p} r_{1}^{p}+\sum_{j=p+1}^{m} \lambda_{j} q_{j}^{\prime}$, $\mathbb{P}=\left\{L, L^{\prime}\right\}$ is not associated with a class of strategically equivalent decision trees because otherwise $\mathbb{P}$ should have include the following 4 lotteries $L, L^{\prime}, L^{\prime \prime}, L^{\prime \prime \prime}$ where $L^{\prime \prime}=\lambda_{1} r_{1}^{1}+$ $\lambda_{2} r_{2}^{2}+\lambda_{3} r_{1}^{3}+\ldots .+\lambda_{p} r_{1}^{p}+\sum_{j=p+1}^{m} \lambda_{j} q_{j}^{\prime}$ and $L^{\prime \prime \prime}=\lambda_{1} r_{2}^{1}+\lambda_{2} r_{1}^{2}+\lambda_{3} r_{1}^{3}+\ldots .+\lambda_{p} r_{1}^{p}+\sum_{j=p+1}^{m} \lambda_{j} q_{j}^{\prime}$. 


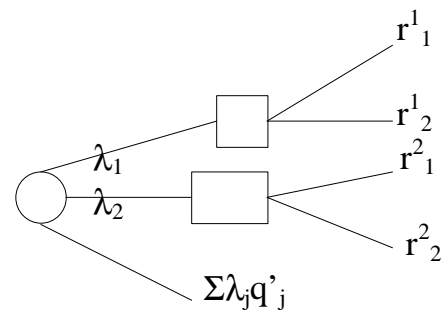

We will show that if we take the decision problems building by taking two elements from $\left\{L_{1}, L_{2}, \ldots, L_{k}\right\}$ then if DM is information averse for these decision problems then he is information averse for the decision problem $\left\{\left\{L_{1}, L_{2}, \ldots ., L_{k}\right\} ; C\right\}$.

Let $\mathbb{P}=\left\{L, L^{\prime}\right\}$ be a decision problem with $L, L^{\prime} \in\left\{L_{1}, L_{2}, \ldots, L_{k}\right\}$ then $L$ and $L^{\prime}$ are different other only one consequence.

If $\mathrm{DM}$ is information averse for the decision problem $\mathbb{P}=\left\{\lambda r+(1-\lambda) q^{\prime}, \lambda q+(1-\lambda) q^{\prime}\right\}$, $\forall \lambda \in] 0,1\left[, \forall\right.$ lotteries $r, q$ and $q^{\prime}$ then according to lemma 4 , DM is information averse for the decision problem $\left.\mathbb{P}=\left\{\lambda r+\sum_{j=2}^{m} \lambda_{j} q_{j}^{\prime}, \lambda q+\sum_{j=2}^{m} \lambda_{j} q_{j}^{\prime}\right\}, \forall \lambda \in\right] 0,1\left[, \forall\right.$ lotteries $r, q$ and $q^{\prime}$. Therefore DM is information for the decision problem $\mathbb{P}=\left\{L, L^{\prime}\right\}$, associated with a class of strategically equivalent decision trees and $L, L^{\prime} \in\left\{L_{1}, L_{2}, \ldots, L_{k}\right\}$.

If DM is information averse for any decision problem $\mathbb{P}=\left\{L, L^{\prime}\right\}$ associated with a class of strategically equivalent decision trees and $L, L^{\prime} \in\left\{L_{1}, L_{2}, \ldots, L_{k}\right\}$ then lemma 1 gives us for any decision problem $\mathbb{P}$ the possible con gurations of preference at the differents decision nodes 1,2 and 3 . We can induce (like in case $\operatorname{Card} \mathbb{O}_{i}=3$ ) the preferences over $\left\{L_{1}, L_{2}, \ldots, L_{k}\right\}$ at nodes 1 and 2 , and the preferences over $\left\{r_{1}^{1}, \ldots, r_{n_{1}}^{1}, r_{1}^{2}, \ldots, r_{n_{2}}^{2}, \ldots \ldots, r_{1}^{p} \ldots, r_{n_{p}}^{p}\right\}$ at nodes 3. [See the above graph called Figure PG]

These preferences are not necessarily complete since some pairs of $\left\{L_{1}, L_{2}, \ldots, L_{k}\right\}$ are not decision problems in the sense of de nition 1.

Moreover some con gurations of preferences at nodes 1, 2 and 3 lead to cyclical preferences. However we do not take such a preference into account in this analysis since we have assume decisiveness of the choice functions (hypothesis H4).

When the displayed preferences do not lead to a cycle then there is a selection (using the dual de nition of rational choice if the exhibited strict preference is not complete) of a lottery $L_{i_{0}} \in\left\{L_{1}, L_{2}, \ldots, L_{k}\right\}$ over $T$ (node 2), of a lottery $L_{j_{0}} \in\left\{L_{1}, L_{2}, \ldots, L_{k}\right\}$ over $T^{\prime}$ (node 3 ) and of a lottery $L_{p_{0}} \in\left\{L_{1}, L_{2}, \ldots, L_{k}\right\}$ at node 1 . We will show from one part that $L_{i_{0}}$ and $L_{j_{0}}$ are unique, and from another part that the lottery $L_{p_{0}}$ selected at decision node 1 is the same as the lottery $L_{i_{0}}$ selected at decision 2 , and nally that $L_{p_{0}} \neq L_{j_{0}}$. This implies that at decision node 1 , DM will prefer to choose over $T$ in order to get $L_{i_{0}}$. Then he is information averse.

Let us show the unicity of $L_{i_{0}}$ (we can show likewise the unicity of $L_{j_{0}}$ using the same strategy of proof): 
Suppose that two lotteries were selected : $L_{i_{0}}$ and $L_{i_{0}}^{\prime}$. Then necessarily $\left\{L_{i_{0}}, L_{i_{0}}^{\prime}\right\}$ is not a decision problem in the sense of de nition 1. Otherwise there will exist a strict preference between $L_{i_{0}}$ and $L_{i_{0}}^{\prime}$ contradicting the assumption that they were both selected.

However there exist two lotteries $L_{i_{0}}^{\prime \prime}$ and $L_{i_{0}}^{\prime \prime \prime}$ with $L_{i_{0}}, L_{i_{0}}^{\prime}, L_{i_{0}}^{\prime \prime}$ and $L_{i_{0}}^{\prime \prime \prime}$ pairwise differents such that $\left\{L_{i_{0}}, L_{i_{0}}^{\prime \prime}\right\},\left\{L_{i_{0}}, L_{i_{0}}^{\prime \prime \prime}\right\},\left\{L_{i_{0}}^{\prime}, L_{i_{0}}^{\prime \prime}\right\}$ and $\left\{L_{i_{0}}^{\prime}, L_{i_{0}}^{\prime \prime \prime}\right\}$ are decision problems in the sense of de nition 1 . Indeed let us, without loss of generality, set $L_{i_{0}}=\lambda_{1} r_{1}^{1}+\lambda_{2} r_{1}^{2}+$ $\lambda_{3} r_{1}^{3}+\ldots .+\lambda_{p} r_{1}^{p}+\sum_{j=p+1}^{m} \lambda_{j} q_{j}^{\prime}$ and $L_{i_{0}}^{\prime}=\lambda_{1} r_{2}^{1}+\lambda_{2} r_{2}^{2}+\lambda_{3} r_{1}^{3}+\ldots .+\lambda_{p} r_{1}^{p}+\sum_{j=p+1}^{m} \lambda_{j} q_{j}^{\prime}$. $\left\{L_{i_{0}}, L_{i_{0}}^{\prime}\right\}$ is not a decision problem because the two lotteries are different over two states of nature. Let us take $L_{i_{0}}^{\prime \prime}=\lambda_{1} r_{1}^{1}+\lambda_{2} r_{2}^{2}+\lambda_{3} r_{1}^{3}+\ldots+\lambda_{p} r_{1}^{p}+\sum_{j=p+1}^{m} \lambda_{j} q_{j}^{\prime}$ and $L_{i_{0}}^{\prime \prime \prime}=\lambda_{1} r_{2}^{1}+\lambda_{2} r_{1}^{2}+\lambda_{3} r_{1}^{3}+\ldots .+\lambda_{p} r_{1}^{p}+\sum_{j=p+1}^{m} \lambda_{j} q_{j}^{\prime}$. It is easy to check that $\left\{L_{i_{0}}, L_{i_{0}}^{\prime \prime}\right\},\left\{L_{i_{0}}, L_{i_{0}}^{\prime \prime \prime}\right\},\left\{L_{i_{0}}^{\prime}, L_{i_{0}}^{\prime \prime}\right\}$ and $\left\{L_{i_{0}}^{\prime}, L_{i_{0}}^{\prime \prime \prime}\right\}$ are decision problems.

Let us continue the proof. Since $L_{i_{0}}$ and $L_{i_{0}}^{\prime}$ are both selected then we have : $L_{i_{0}}$ $\succ_{2} L_{i_{0}}^{\prime \prime}, L_{i_{0}} \succ_{2} L_{i_{0}}^{\prime \prime \prime}, L_{i_{0}}^{\prime} \succ_{2} L_{i_{0}}^{\prime \prime}, L_{i_{0}}^{\prime} \succ_{2} L_{i_{0}}^{\prime \prime \prime}$. These preferences necessarily exist since $\left\{L_{i_{0}}, L_{i_{0}}^{\prime \prime}\right\},\left\{L_{i_{0}}, L_{i_{0}}^{\prime \prime \prime}\right\},\left\{L_{i_{0}}^{\prime}, L_{i_{0}}^{\prime \prime}\right\}$ and $\left\{L_{i_{0}}^{\prime}, L_{i_{0}}^{\prime \prime \prime}\right\}$ are decision problems.

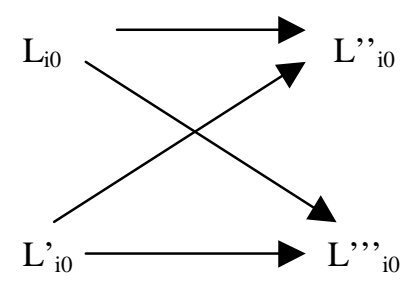

Well according to lemma 1 , at node 3 for the decision problem $\left\{L_{i_{0}}, L_{i_{0}}^{\prime \prime \prime}\right\}$, we have $r_{2}^{1} \succ_{3} r_{1}^{1}$ (because $\left.L_{i_{0}} \succ_{2} L_{i_{0}}^{\prime \prime \prime}\right)$ and at node 3 for the decision problem $\left\{L_{i_{0}}^{\prime}, L_{i_{0}}^{\prime \prime}\right\}$, we have $r_{1}^{1} \succ_{3} r_{2}^{1}$ (because $L_{i_{0}}^{\prime} \succ_{2} L_{i_{0}}^{\prime \prime}$ ). And that contradicts the asymmetry of $\succ_{3}$.

Let us complete the PART I proof by showing that $L_{p_{0}} \neq L_{j_{0}}$ and $L_{p_{0}}=L_{i_{0}}$.

Obviously, $L_{p_{0}} \neq L_{j_{0}}$ because according to lemma 1 , preferences at decision nodes 3 and 1 do not coincide.

Obviously, $L_{p_{0}}=L_{i_{0}}$ because according to lemma 1, preferences at decision nodes 2 and 1 coincide.

PART II : There is no lost of generality when restricting the analysis to $C_{1}^{*}$ and $C_{2}^{*}$.

Let us remark that if the lotteries are n-stages in the sense de ned in PART I, then there are exactly $\mathrm{n}$ classes $C_{t}^{*}$. $C_{1}^{*}$ includes the tree $T$ described in Figure PG and $C_{2}^{*}$ includes the tree $T^{\prime}$ described in gure PG. We want to show that $(* *) \Longrightarrow\left[\forall \mathfrak{C}_{i}\right.$, $\left.\forall T \in C_{t}^{*}, \forall T^{\prime} \in C_{t^{\prime}}^{*}, t>t^{\prime} \Longrightarrow T P_{R_{i}} T^{\prime}\right]$. Let us rename by $T 1$ the tree belonging to $C_{1}^{*}$ and by $T 2$ the tree belonging to $C_{2}^{*}$. Let $T$ belongs to $C_{3}^{*}$ then $I(T)=2$. This tree $T$ was built from $T 2$ by the following way: over one $j_{0}$, we have, 


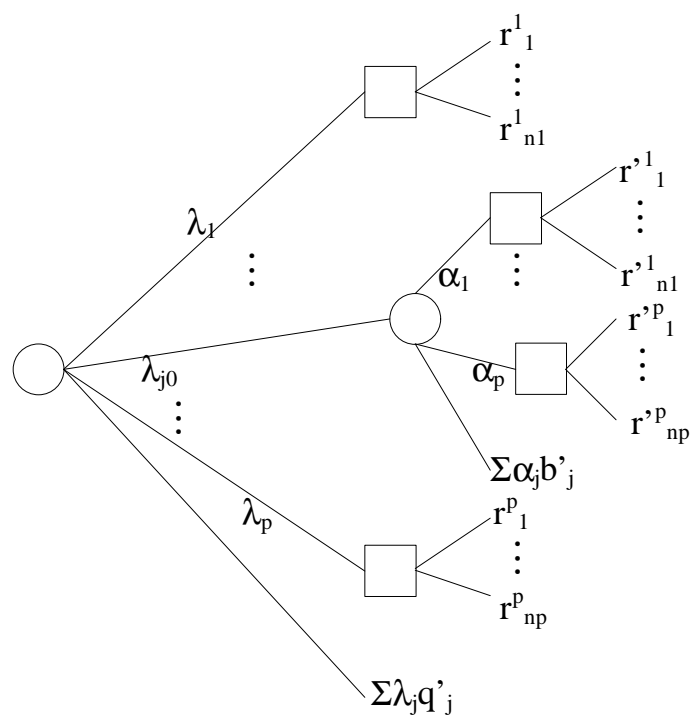

Let us call $\tau$ the following decision tree :

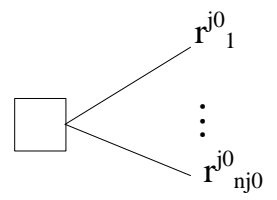

and let us call $\tau^{\prime}$ the below decision tree :

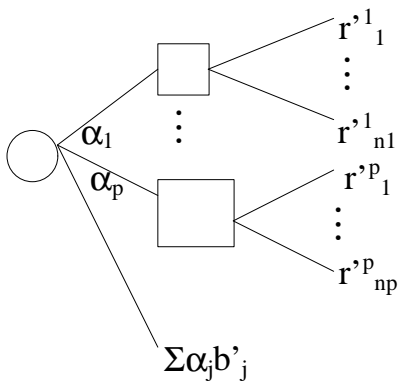

$\tau$ and $\tau^{\prime}$ are strategically equivalent. Moreover $I(\tau)=0$ and $I\left(\tau^{\prime}\right)=1 . \quad \tau$ and $\tau^{\prime}$ belong to the class of strategically equivalent decision trees $\mathfrak{C}^{\prime}$ which is associated to the 
following decision problem $\mathbb{P}^{\prime}=\left\{r_{1}^{j 0}, r_{2}^{j 0}, \ldots . ., r_{n_{j 0}}^{j 0}\right\}$. According to PART I , we have $\tau$ $P_{R^{\prime}} \tau^{\prime}$ where $P_{R^{\prime}}$ is the asymetric part of $R^{\prime}$ the DM s preference relation over $\mathfrak{C}^{\prime}$. Let us now remind that $T 2$ and $T$ are:

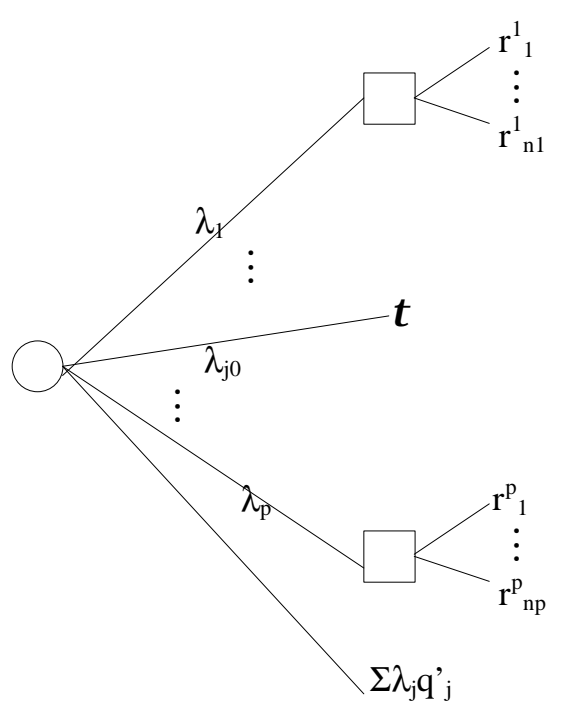

Figure T2

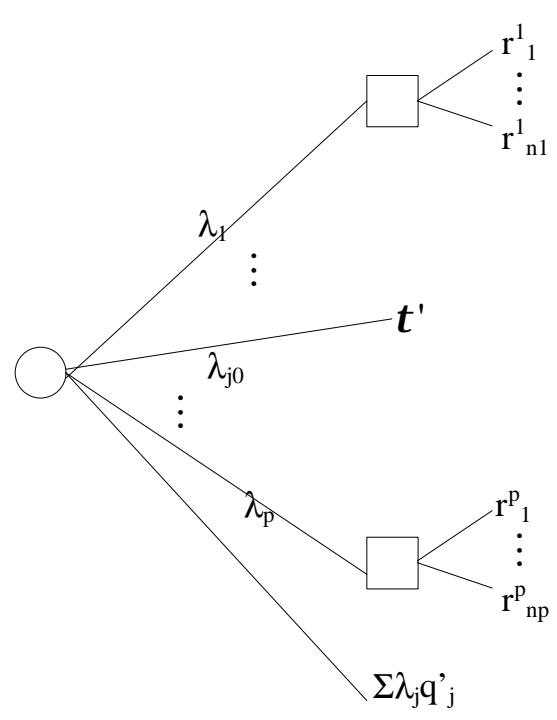

Figure T 
Using H3, we have $\tau P_{R^{\prime}} \tau^{\prime} \Longrightarrow T 2 P_{R} T, \forall T \in C_{3}^{*}$. Likewise, one can show that $T P_{R} T^{\prime}, \forall T \in C_{t-1}^{*}, T^{\prime} \in C_{t}^{*}, t=2$ to $n$. We nally use transitivity of $P_{R}$ to get the required result : $T_{1} P_{R} T_{2} P_{R} \ldots \ldots P_{R} T_{n}$.

\section{References}

[1] Allais, M. (1953), Le comportement de 1 homme rationnel devant le risque: Critique des postulats et Axiomes de l école Américaine , Econometrica, Volume 21, pp. 503546.

[2] Blackwell, D. (1953), Equivalent Comparisons of Experiments , Annals of Mathematical Statistics, June, pp.265-272.

[3] Brocas, I. \& Carrillo, J.D. (2000), The Value of Information when Preferences are Dynamically Inconsistent , European Economic Review, Papers and Proceedings, Volume 44, pp.1104-1115.

[4] Caillaud, B. \& Jullien, B. (2000), Modelling Time Inconsistent Preferences , European Economic Review, Papers and Proceedings, Volume 44, pp. 1116-1124.

[5] Datta, M.; Mirman, L.J. \& Schlee E.E. (2000), Optimal Experiment in Signal Dependent Decision Problems, forthcoming in International Economic Review.

[6] Grant, S., Kajii., A. \& Polak, B. (1998), Intrinsic Preference for Information , Journal of Economic Theory, Volume 83, n², pp.233-259.

[7] Jensen, N. E. (1967), An Introduction to Bernouillan Utility Theory I: Utility Function , Swedish Journal of Economics, Volume 69, pp. 163-183.

[8] Kahneman, D. \& Tversky, A. (1979), Prospect Theory: An Analysis of Decision under Risk , Econometrica, Volume 47, pp. 263-291.

[9] Karni, E. \& Schmeidler, D. (1990), Utility Theory with Uncertainty , in W. Hildenbrand and H. Sonnenschein eds, Handbook of Mathematical Economics, Volume 4, Chapter 33.

[10] Karni, E. \& Schmeidler, D. (1991), Atemporal Dynamic Consistency and Expected Utility , Journal of Economic Theory, Volume 54, pp. 401-408.

[11] Kreps, D.M. \& Porteus, E.L. (1978), Temporal resolution of uncertainty and dynamic choice theory , Econometrica, Volume 46, pp. 565-577.

[12] Kreps, D.M. \& Porteus, E.L. (1979), Temporal von Neumann-Morgenstern and Induced Preferences , Journal of Economic Theory, Volume 20, pp. 81-109.

[13] LaValle, I. \& Fishburn, P.C. (1987), Equivalent Decision trees and their associated strategy sets , Theory and Decision, Volume 23, pp. 37-63. 
[14] LaValle, I.H. (1978), Fundamentals of Decisions Analysis, Holt, Rinehart \& Winston Inc., NY.

[15] Lerman, C.; Narod, S; Schulman, K.\& al. (1996), BRCA1 Testing in Families with Hereditary Breast-Ovarian Cancer , Journal of the American Medical Association, Volume 275, pp. 1885-1892.

[16] Machina, M.J. (1982), Expected utility analysis without the independence axiom , Econometrica, Volume 50, pp. 277-323.

[17] Machina, M.J. (1989), Dynamic consistency and non-expected utility models of choice under uncertainty , Journal of Economic Litterature, Volume 27, pp. 16221668 .

[18] Raiffa, H. (1968), Decision Analysis: Introductory Lectures on Choices Under Uncertainty, Addison-Wesley Publishing Co, Reading, MA.

[19] Richter, K. (1971), Rational Choices , in J.S. Chipman, L. Hurwicz, K. Richter, and H. Sonnenschein eds, Preference, Utility and Demand, Harcourt Brace Jovanovitch, New York, pp. 29-58.

[20] Savage, L.J. (1954), The Foundations of Statistics, Dover, New York.

[21] Schlee, E.E. (1990), The value of Information in Anticipated Utility Theory , Journal of Risk and Uncertainty, Volume 3, pp. 83-92.

[22] Schlee, E.E. (2001), The Value of Information in Efficient Risk Sharing Arrangements , American Economic Review, Volume 91, pp. 509-524.

[23] Von Neumann, J. \& Morgenstern, O. (1947), Theory of Games and Economic Behavior, 2nd rev. ed., Princeton University Press, Princeton.

[24] Wakker, P. (1988), Nonexpected Utility as Aversion of Information , Journal of Behavioral Decision Making, Volume 1, pp. 169-175.

[25] Wakker, P. (1999), Justifying Bayesianism by Dynamic Decision Principles , Working Paper, Leiden University Medical Center, January, 19 pages.

[26] Yaari, M.E. (1985), On the Role of Dutch Books In the Theory of Choice Under Risk , Nancy Schwartz Memorial Lecture, J.L. Kelloggs Graduate School of Management, Northwestern University. 